\title{
Stability of stationary endwall boundary layers during spin-down
}

\author{
By J. M. LOPEZ $Z^{1}$ AND P. D. WEIDMAN ${ }^{2}$ \\ ${ }^{1}$ Department of Mathematics and Earth System Science Center, The Pennsylvania State \\ University, University Park, PA 16802, USA \\ ${ }^{2}$ Department of Mechanical Engineering, University of Colorado, Boulder, CO 80302, USA
}

(Received 18 January and in revised form 14 June 1996)

Since Bödewadt's (1940) seminal work on the boundary layer flow produced by a fluid in solid-body rotation over a stationary disk of infinite radius there has been much interest in determining the stability of such flows. To date, it appears that there is no theoretical study of the stability of Bödewadt's self-similar solution to perturbations that are not self-similar. Experimental studies have been compromised due to the difficulty in establishing these steady flows in the laboratory. Savaş (1983, 1987) has studied the endwall boundary layers of flow in a circular cylinder following impulsive spin-down. During the first few radians of rotation, the endwall boundary layers have a structure very similar to Bödewadt layers. For certain conditions, Savaş has observed a series of axisymmetric waves travelling radially inwards in the endwall boundary layers. The conjecture is that these waves represent a mode of instability of the Bödewadt layer. Within a few radians of rotation however, the centrifugal instability of the sidewall layer dominates the spin-down process and the endwall waves are difficult to examine further.

Here, the impulsive spin-down problem is examined numerically for Savaş' (1983, 1987 ) conditions and good agreement with his experiments is achieved. New experimental results are also presented, which include quantitative space-time information regarding the axisymmetric waves. These agree well with both the numerics and the earlier experimental work. Further, a related problem is considered numerically. This flow is also initially in solid-body rotation, but only the endwalls are impulsively stopped, keeping the sidewall rotating. This results in a flow virtually identical to the usual spin-down flow for the first few radians of rotation, except in the immediate vicinity of the sidewall. The sidewall layer is no longer centrifugally unstable and the circular waves on the endwalls are observed without the influence of the sidewall instability.

\section{Introduction}

Bödewadt's (1940) similarity solution for the boundary layer flow over a stationary disk of infinite extent produced by fluid in solid-body rotation above provides an exact solution of the Navier-Stokes equations. The solution, which has been the subject of much controversy (Greenspan 1968), depends only on the similarity variable $z$, the direction normal to the disk. The full three-dimensional boundary layer flow however, depends on $z$ and $r$, the radial coordinate ( $\vartheta$ as well if azimuthal symmetry is broken). Bödewadt's (1940) is not the only solution of this type. Earlier, von Kármán (1921) 
presented an exact solution of the Navier-Stokes equations for the flow produced by the constant rotation of a disk of infinite extent. The controversy with the Bödewadt solution is that it requires inflow from infinity to approach similarity in the boundary layer as $r \rightarrow 0$. The speculation in the literature (Moore 1956; Rogers \& Lance 1964) has been that this boundary layer flow may 'break down' at some finite radius and hence Bödewadt's (1940) similarity solution may not be achieved in practice. There is little solid evidence supporting or refuting this conjecture in the literature.

Virtually all the theoretical and numerical studies are in terms of the boundary layer equations (using similarity variables) and the problem of matching the boundary layer flow to the external flow has not been satisfactorily resolved in these formulations. There have been some experimental studies, using finite disks, but producing the external flow in solid-body rotation is non-trivial and the results are inconclusive. Rott \& Lewellen (1966) give an extensive review of these works. Schwiderski \& Lugt (1964) conjecture, based on a low-order expansion in $r$ of the steady Navier-Stokes equations, that the solution of Bödewadt (1940) would not be stable due to an inflection point instability beyond the Reynolds number where the boundary layer profiles first show spatial oscillations.

Savaş $(1983,1987)$ conducted a series of experiments where initially a regime corresponding to quasi-steady Bödewadt flow exists. The initial state consists of a filled circular cylinder in solid-body rotation of angular rotation rate $\Omega$. At time $t=0$, the cylinder is impulsively stopped. Apart from thin boundary layers on all rigid surfaces, the interior flow continues to be in solid-body rotation for some time. The stationary endwalls of the cylinder are the finite analogue of Bödewadt's disk of infinite extent. Until secondary motions grow, Savaş $(1983,1987)$ suggests that this flow provides a means to examine the stability of Bödewadt's (1940) solution. For a wide range of Reynolds numbers $\left(R e=\Omega R^{2} / v\right.$, where $R$ is the cylinder radius and $v$ is the kinematic viscosity), Savaş (1987) observed that in some mean sense, the profile of the boundary layer in his spin-down experiments matched Bödewadt's (1940) profiles (at least for about one rotation period). He also observed that for $R e \approx 10^{4}$, the boundary layer supported a series of circular (i.e. axisymmetric) waves which first become apparent at a radius $r \approx R / 2$ and propagate radially inwards, but do not reach the centre $(r=0)$. In the vicinity of the axis, the boundary layer is of Bödewadt type. Based on a series of these spin-down experiments, Savaş (1987) suggests that the Bödewadt (1940) solution loses stability to these circular waves at a local Reynolds number $r_{w}(\Omega / v)^{1 / 2} \approx 25$, where $r_{w}$ is the radius at which the waves are observed. (Note, this local Reynolds number is the square root of the usual form for a Reynolds number.) The value 25 corresponds to the smallest radius at which the waves, i.e. bright circles in the flow visualizations, were observed, with small $\Omega$.

In order to investigate the stability of the similarity solution of Bödewadt (1940), one must allow for the growth of disturbances that depend on the radial coordinate. Azimuthal variations are also possible; however Savaş' $(1983,1987)$ experiments suggest that the endwall layers first become unstable to axisymmetric circular waves as $R e$ is increased, observing spiral modes only at Re much larger than those at which the circular waves were observed. Once disturbances with radial variations are allowed, a radial length scale must be introduced. The similarity form of Bödewadt has no radial length scale. The flow is then not completely specified until the conditions on the axis and at large radius are specified, and herein lies the problem for studying the stability of the similarity solution. If one attempts to specify conditions at $R=\infty$, then solid-body rotation of the interior has $v \rightarrow \infty$ which is unphysical. The only alternative is for $R \neq \infty$, i.e. to consider the case of a finite disk in a finite domain. 
This then has implications for the role of the interaction between the primary and secondary flows.

Problems associated with studying the stability of steady Bödewadt flows using the quasi-steady system provided for by the early-time spin-down experiments are the following. (i) The transient nature of the flow means that conclusions regarding the long-time stability of the flow are difficult to make. Are the waves a transient inertial oscillation following the impulsive stopping of the cylinder? It should be noted however, that the experiments of Savas (1987) provide strong evidence that the inertial modes of the cylinder do not play a significant direct role in the development of the circular waves in the Bödewadt layer. (ii) During spin-down, the sidewall boundary layer is centrifugally unstable giving rise to Taylor-Görtler (T-G) cells (Maxworthy 1971; Weidman 1976b; Neitzel \& Davis 1980, 1981; Valentine \& Miller 1994). These grow to finite amplitude in about one rotation period, depending on the size of the perturbation to the layer, and subsequently completely dominate the flow. Are the waves observed by Savaş $(1983,1987)$ due to the centrifugal instability of the sidewall layer interacting with the endwall layer?

In order to address these issues, a series of numerical and laboratory experiments have been performed. One series reproduces as closely as possible the spin-down experiments of Savaş $(1983,1987)$, both numerically and in the laboratory. Another series of numerical experiments incorporates a modification to the spin-down experiment which eliminates the centrifugal instability and allows for non-trivial long-time fluid motion. Of course, for spin-down in a rigid cylinder, as in the experiments of Savaş, the fluid eventually comes to rest.

\section{Governing equations}

The equations governing the flow are the axisymmetric Navier-Stokes equations, together with the continuity equation and appropriate boundary and initial conditions. It is convenient to write these using a cylindrical polar coordinate system $(r, 9, z)$, relative to a stationary observer with the origin at the centre of the cylinder and the positive- $z$ axial direction being towards the top endwall. For axisymmetric flow, there exists a Stokes streamfunction $\psi$ and the velocity vector in cylindrical polars is

$$
\boldsymbol{u}=\left(-\frac{1}{r} \psi_{z}, v, \frac{1}{r} \psi_{r}\right)
$$

Subscripts denote partial differentiation with respect to the subscript variable. This form of the velocity automatically satisfies the continuity equation. It is also convenient to introduce a new variable, the angular momentum $\Gamma=r v . \Gamma$ is proportional to the circulation. The vorticity field corresponding to (2.1) is

$$
\omega=(\xi, \eta, \zeta)=\left(-\frac{1}{r} \Gamma_{z},-\frac{1}{r} \nabla_{*}^{2} \psi, \frac{1}{r} \Gamma_{r}\right)
$$

where

$$
\nabla_{*}^{2}=()_{z z}+()_{r r}-\frac{1}{r}()_{r}
$$

The velocity and vorticity fields can be decomposed into azimuthal $(\vartheta)$ and meridional $(m)$ fields, where

$$
\boldsymbol{u}^{\vartheta}=\left(0, \frac{1}{r} \Gamma, 0\right), \boldsymbol{\omega}^{\vartheta}=\left(0,-\frac{1}{r} \nabla_{*}^{2} \psi, 0\right), \boldsymbol{u}^{m}=\left(-\frac{1}{r} \psi_{z}, 0, \frac{1}{r} \psi_{r}\right), \boldsymbol{\omega}^{m}=\left(-\frac{1}{r} \Gamma_{z}, 0, \frac{1}{r} \Gamma_{r}\right) ;
$$


so $\boldsymbol{\omega}^{\vartheta}=\boldsymbol{\nabla} \times \boldsymbol{u}^{m}$ and $\boldsymbol{\omega}^{m}=\boldsymbol{\nabla} \times \boldsymbol{u}^{\vartheta}$. Further, $\Gamma$ plays the role of a streamfunction for the meridional vorticity field (Bragg \& Hawthorne 1950). In other words, contours of $\Gamma$ in a meridional plane are cross-sections of vortex surfaces (vortex lines), just as contours of $\psi$ are cross-sections of streamsurfaces (streamlines). These give the local direction of the vorticity and velocity vectors in the plane, respectively, and the azimuthal components of the vectors give the degree to which the vectors are directed out of the plane.

The axisymmetric Navier-Stokes equations, in terms of $\psi, \Gamma$ and $\eta$, are

$$
\begin{gathered}
\mathrm{D} \Gamma=\nabla_{*}^{2} \Gamma / \operatorname{Re}, \\
\mathrm{D}(\eta / r)=\left\{\nabla^{2}(\eta / r)+\frac{2}{r}(\eta / r)_{r}\right\} / \operatorname{Re}+\left(\Gamma^{2} / r^{4}\right)_{z},
\end{gathered}
$$

where

$$
\left.\begin{array}{rl}
\nabla_{*}^{2} \psi & =-r \eta \\
\mathrm{D} & =()_{t}-(1 / r) \psi_{z}()_{r}+(1 / r) \psi_{r}()_{z}, \\
\nabla^{2} & =()_{z z}+()_{r r}+(1 / r)()_{r},
\end{array}\right\}
$$

and $R e=\Omega R^{2} / v$. The length scale is the radius of the cylinder $R$ and the time scale is $1 / \Omega$. The other governing non-dimensional parameter is the cylinder aspect ratio $H / R, H$ being the cylinder height.

Equation (2.3) shows that the inertial change in the azimuthal vorticity, and hence a source of meridional flow, is driven by axial gradients in the angular momentum. The physical origin (Davidson 1989) of the source term on the right-hand side of (2.3) is the azimuthal component of $\nabla \times\left(u^{9} \times \omega^{m}\right)$. It corresponds to the turning of meridional vorticity into the azimuthal direction by the azimuthal velocity. This turning of the vorticity vector is responsible for the particular structure of the endwall boundary layer being investigated.

\subsection{Computational technique}

The governing equations are discretized using second-order centred differences to approximate all spatial derivatives. Trial computations have shown that $Z_{2}$ symmetry (reflection about the cylinder half-height) is only broken following the growth of the Taylor-Görtler cells to finite amplitude in the spin-down case, and it is observed not to be broken for the range of parameters considered here in the modified flow. Consequently, all computed results presented correspond to flows with $Z_{2}$ symmetry imposed. The finite difference grid is stretched in the $z$-direction in order to better resolve the endwall boundary layer. Although the T-G instability of the sidewall layer results in an axial periodic structure that may be affected by the coordinate stretching, sufficient resolution is employed for this not to be a concern. The grid is uniform in the $r$-direction as waves with large radial gradients travel in the radial direction across most of the radius, and hence there is no preferred radial location where stretching would be beneficial. The axial stretching is accomplished by

$$
z=\left(z^{\prime}+a \sin \left(\pi z^{\prime}\right)\right) H / 2 R,
$$

where $z^{\prime}$ varies uniformly over the interval $[0,1]$ and the stretching factor $a=0.1$ has been used for all the results presented here. The top endwall is located at $z=H / 2 R$ and $z=0$ is the location of the cylinder half-height. The number of grid points in the radial and axial directions for the half-cylinder are $n r$ and $n z$.

The axis, reflection, and boundary conditions are, for both the spin-down and modified flows: (i) on the axis ( $r=0), \Gamma=\eta=\psi=0$; (ii) at the cylinder half-height 
$(z=0), \Gamma_{z}=\eta=\psi=0$; (iii) on the top endwall $(z=H / 2 R), \Gamma=\psi=0$ and $\eta=-\psi_{z z} / r$; (iv) on the sidewall $\left(r=1\right.$ ), $\psi=0$ and $\eta=-\psi_{r r}$; however the boundary condition for $\Gamma$ differs between the two cases: $\Gamma=0$ for spin-down and $\Gamma=1$ for the modified flow.

The derivative boundary conditions are approximated by one-sided differences. The reflection condition at $z=0$, in discrete form, is

$$
\Gamma_{1, i}=\left(4 \Gamma_{2, i}-\Gamma_{3, i}\right) / 3
$$

and is third-order accurate. The boundary conditions on $\eta$ are obtained by expanding $\psi$ to third order as Taylor series about the first and second grid points in from the boundaries and eliminating the third-derivative terms between them, noting that $\psi$ and its first derivatives vanish at the boundaries. This results in second-order-accurate estimates for $\psi_{r r}$ and $\psi_{z z}$ on the sidewall and endwall respectively. The discrete $\eta$ conditions are then

and

$$
\eta_{j, n r}=-\left(8 \psi_{j, n r-1}-\psi_{j, n r-2}\right) / 2 \delta r^{2}
$$

$$
\eta_{n z, i}=-\left(8 \psi_{n z-1, i}-\psi_{n z-2, i}\right) /\left(2 r_{i}\left(\delta z^{\prime} \mathrm{d} z / \mathrm{d} z^{\prime}\right)^{2}\right)
$$

where $\delta r=1 /(n r-1)$ and $\delta z^{\prime}=1 /(n z-1)$.

The discrete versions of (2.2) and (2.3) are integrated using a second-order predictor-corrector scheme. Writing (2.2) and (2.3) as

$$
\Gamma_{t}^{n}=G_{1}\left(\Gamma^{n}, \eta^{n}, \psi^{n}\right)
$$

and

$$
\eta_{t}^{n}=G_{2}\left(\Gamma^{n}, \eta^{n}, \psi^{n}\right)
$$

where $G_{1}$ and $G_{2}$ represent nonlinear operators following the second-order central differencing of the equations and the superscript $n$ denotes the $n$th time level $t=n \delta t$. The predictor-corrector algorithm entails the following stages, starting from known values $\Gamma^{n}, \eta^{n}$, and $\psi^{n}$ :

(a) Evaluate $\Gamma^{\star}=\Gamma^{n}+0.5 \delta t G_{1}\left(\Gamma^{n}, \eta^{n}, \psi^{n}\right)$ and $\eta^{\star}=\eta^{n}+0.5 \delta t G_{2}\left(\Gamma^{n}, \eta^{n}, \psi^{n}\right)$ on the interior grid points.

(b) Solve $\nabla_{*}^{2} \psi^{\star}=-r \eta^{\star}$ using generalized cyclic reduction (Sweet 1974).

(c) Implement boundary conditions on $\Gamma^{\star}, \eta^{\star}$, and $\psi^{\star}$.

(d) Evaluate $\Gamma^{n+1}=\Gamma^{n}+\delta t G_{1}\left(\Gamma^{\star}, \eta^{\star}, \psi^{\star}\right)$ and $\eta^{n+1}=\eta^{n}+\delta t G_{2}\left(\Gamma^{\star}, \eta^{\star}, \psi^{\star}\right)$ on the interior grid points.

(e) Solve $\nabla_{*}^{2} \psi^{n+1}=-r \eta^{n+1}$ using generalized cyclic reduction.

(f) Implement boundary conditions on $\Gamma^{n+1}, \eta^{n+1}$, and $\psi^{n+1}$.

All the computations are started impulsively from a state of solid-body rotation, i.e. $\psi=\eta=0$ and $\Gamma=r^{2}$.

All of the results presented were computed on grids of $n r \times n z=301 \times(1+300 H / 2 R)$. This resolution was found to be more than adequate for the largest $R e$ considered, and the low-Re cases were over-resolved. A time step $\delta t=0.01$ was used in most cases. All computations were performed using 64-bit arithmetic.

\section{Spin-down in a finite-length cylinder}

Neitzel \& Davis (1981) report on computations of impulsive spin-down in a finite-length cylinder for $R e$ up to $4 \times 10^{3}$. However, their study concentrated on the sidewall centrifugal instability and did not address any possible instabilities in 
the endwall layers. Numerical evidence from the present computations of circular waves at $R e \approx 10^{3}$ will be presented. Valentine \& Miller (1994) have also presented computations of impulsive spin-down in a finite-length cylinder for $\operatorname{Re}$ up to $4 \times 10^{3}$. They mention the existence of a 'solitary wave' that propagates from the endwall to the midplane along the central axis, but do not identify any circular waves in the endwall layers.

It is instructive to consider the flow in terms of the vortex lines. Initially, the fluid is in solid-body rotation with the cylinder. The vortex lines are everywhere in the axial direction, i.e. $\Gamma=\Gamma(r)=r^{2}$. In the present coordinate system (\$2.1), the vortex lines all emanate from the bottom endwall and terminate at the top endwall. When the cylinder is stopped impulsively at $t=0$, the vortex lines can no longer begin or end on the now stationary endwalls. Also, on the sidewall $(r=1) \Gamma=0$ for $t>0$ while the interior flow is still in solid-body rotation with $\Gamma=r^{2}$. Thus the vortex lines are bent radially outwards from the axial direction in the thin endwall boundary layers formed following the impulsive stop. The radial bending of the vortex lines produces axial gradients in $\Gamma$, and as a consequence of the source term in (2.3), any bending of the vortex lines into the radial direction must also be accompanied by a bending into the azimuthal direction. In the vicinity of $r=1$, the vortex lines in the endwall layers are again bent, this time into the axial direction within a thin sidewall layer where the vortex lines from the top and bottom layer connect. This bending of the vortex lines occurs for all $R e$. At early times following the impulsive stop, $R e$ only determines the thickness of the wall layers into which the vortex lines are bent. The positive and negative axial gradients in $\Gamma$ in the bottom and top endwall layers respectively give rise to azimuthal components of vorticity there inducing radial inflow in these boundary layers. Consequently, the flow, initially consisting of a purely azimuthal velocity and a purely axial vorticity (the primary flow, with $\psi=0$ everywhere), acquires an azimuthal component of vorticity following the impulsive stop, due to vortex line bending.

The azimuthal component of vorticity induces a meridional velocity (the secondary flow, with non-zero $\psi$ ), consisting of two recirculation cells with radial inflow along the endwall layers, flow into the interior from the endwall layers, radial flow from the interior into the sidewall layer, from where the flow is returned to the endwall layers. If this secondary meridional flow is strong enough (as measured by $R e$ ), then significant nonlinear interactions between the primary and the secondary flow are possible. It is these interactions that are addressed here.

One of these interactions occurs in the endwall layers and leads to a boundary layer with spatial oscillations - a Bödewadt-type boundary layer. For sufficiently large $R e$ $\left(O\left(10^{3}\right)\right.$ or larger), the radial inflow in the layers will tend to carry fluid with angular momentum $\Gamma=r v$ from large to smaller radii, essentially pulling the vortex lines in the layer radially inwards (within the layers, near the endwalls, the vortex lines lie essentially parallel to the endwalls). Thus, $\Gamma$ increases in the direction normal to the endwall, inducing radial inflow, and then decreases, inducing radial outflow. This outflow pulls the vortex lines back to larger radii causing another change of sign in the axial gradients of $\Gamma$. In this way, a multi-layered boundary layer structure is built up with the characteristic spatial oscillations of the Bödewadt profile. This development of the boundary layer is described in detail in $\$ 6.1$ and illustrated in figure 5. The structure of the boundary layer at this time is not of similarity form: it is not independent of the radial coordinate as the layer supports waves. The observed structure of the waves from the numerics agrees with Savaş' $(1983,1987)$ description of the circular waves he observed. Below, more detailed comparisons between the 
computed flows and the experimental flows are presented along with details of the structure of the boundary layers and the circular waves.

While this study is primarily concerned with the structure of the endwall layers and the circular waves they support, it is important to be reminded of the other phenomena present during the impulsive spin-down. One such phenomenon that has attracted much attention is due to the sidewall layer being potentially centrifugally unstable during some part of the spin-down evolution. Neitzel \& Davis (1980) describe in detail how the sidewall layers during spin-down may be subject to 'transient' instability (Davis 1976), since the flow is initially globally stable (solid-body rotation) and evolves to another globally stable state (rest). During the spin-down, the angular momentum in the sidewall layer decreases in the radial direction, allowing for the possible growth of T-G cells. Whether these cells grow to finite amplitude, and if so, when their onset takes place and with what wavelength, depends on Re and how large a perturbation the sidewall layer is subjected to. The wavelength of the T-G cells varies with the sidewall layer thickness, and the sidewall layer is continuously becoming thicker due to horizontal diffusion of vorticity (prior to the onset of T-G cells). So, two spin-downs differing only in the size of the perturbations will have different onset times for T-G cells, and since the sidewall layers are of different thicknesses at these times, the wavelengths of the $T-G$ cells will also be different.

The susceptibility of the sidewall layer to the growth of T-G cells is present in cylinders of both finite and infinite length; however the number and parity of the cells clearly depends on the length. Another phenomenon present during spin-down, that is not present in cylinders of infinite length and is independent of the stability state of the sidewall layer, is the formation of corner ring vorticies at the junctions between the endwalls and the sidewall. Their formation is not related to questions of stability, but rather is due to a combination of the bending of vortex lines around the corners, and the horizontal diffusion of vorticity transporting the vortex lines in the sidewall layer radially inwards. This leads, locally in the corner region, to negative $\Gamma_{z}$ near the top endwall, then a small region of positive $\Gamma_{z}$, followed by essentially zero $\Gamma_{z}$ in the sidewall layer further away from the top endwall. This localized region of alternating sign in $\Gamma_{2}$ results in azimuthal vorticity in the form of a corner vortex. The same process occurs in the bottom corner. For Re greater than about $2.5 \times 10^{3}$, the process occurs within the first rotation period. The process depends on $R e$ (see \$6) as it relies in part on the horizontal translation of the vortex lines due to the thickening of the sidewall layer by viscous diffusion. Once formed, the corner vorticies are ingested in the endwall layer by the radial inflow in the region of the endwall layer closest to the endwall.

One implication of the presence of corner vorticies for the study of the stability of Bödewadt type layers in a spin-down experiment is that unlike the effects of the T-G instability of the sidewall, which could be delayed to some extent by performing experiments with very low levels of perturbations (see Mathis \& Neitzel 1985), the corner vorticies are formed by the kinematics of the flow and their formation cannot be controlled by controlling the noise level in an experiment.

\section{Impulsive spin-down experiment}

An experiment was performed to monitor the trajectory of circular disturbances on one endwall of a fluid-filled cylinder brought suddenly to rest from an initial state of solid-body rotation. We adapted the Plexiglas cylinder used in the spin-down experiments of Weidman (1976b). The thin ring bearing supports were left intact, 
but both fitted glass endwalls were removed and the end surfaces of the cylinder were re-machined accurately parallel. One end of the cylinder was then fitted with an aluminium plug designed to accommodate a direct-drive synchronous stepping motor driven by a Superior Electric Slo-Syn Indexer operating at 200 steps/revolution. The opposite end of the cylinder was retrofitted with a clear Plexiglas plug gently scribed on the fluid side with a target pattern of cross-hairs centred on five concentric circles. The lines were made visible by rubbing black waterproof ink into the etched target pattern. The modified cylinder had an average inner diameter $15.12 \mathrm{~cm}$ with run-out $\pm 0.003 \mathrm{~cm}$ over the cylinder length of $26.3 \mathrm{~cm}$ giving an aspect ratio $H / R=3.48$. The opposite endwalls were measured to be flat and parallel within $\pm 0.002 \mathrm{~cm}$ over the diameter.

Data were taken with a digital video camera at 30 frames $^{-1}$ and recorded on a magnetic disk. Reflection patterns from water seeded with a small amount of Kalliroscope were used to identify the unsteady circular waves. Adequate uniform lighting was provided by a $30 \mathrm{~cm}$ diameter $32 \mathrm{~W}$ circular fluorescent tube mounted a short distance away and concentric with the transparent endwall. The video recorder looked through the lighting system and was located about $1 \mathrm{~m}$ away along the axis of the cylinder so that the endwall filled the field of view. The cylinder was rotated at constant angular speed set by the indexer and was measured by interrupting a light encoder once per revolution with a metal shim attached to the perimeter of the cylinder. After rotating at $2.39 \mathrm{rad} \mathrm{s}^{-1}$ for half an hour to achieve solid-body rotation using spin-up time estimates from Weidman (1976a), the motor indexer was stopped. Observation of the rotating cross-hairs in the video recording showed that the lightweight Plexiglas cylinder stopped within $1 / 30 \mathrm{~s}$. This then identified the time origin. The fluid temperature recorded before the run was $21.8^{\circ} \mathrm{C}$ corresponding to a kinematic viscosity of $0.00962 \mathrm{~cm}^{2} \mathrm{~s}^{-1}$ for pure water. No correction for fluid density or viscosity was made for the small amount of Kalliroscope added for flow visualization or for the slight elevation in water temperature that may have taken place during spin-up. The Reynolds number for this impulsive spin-down experiment was $R e=1.397 \times 10^{4}$.

Analysis of 25 individual frames during the short period of non-dimensional time $3<t<10$ of circular wave evolution was performed on a computer. Circular waves appeared as bright circles superimposed on a darker matted background. A priori identification of these bright circles with a particular part of the wave pattern is difficult (cf. Savaş 1985; Weidman 1989), but they most certainly represent regions in the unsteady flow where the Kalliroscope flakes are actively rotating, i.e. regions of relatively high azimuthal vorticity. The average diameters of identifiable circular disturbances were measured by clicking a movable cursor on the bright circles intersected by a cross-hair and recording pixel positions. Calibration of distances between pixel positions was easily performed using the inscribed concentric circles of known diameters. Though somewhat subjective, we also measured the average diameter of the debris boundary defined as the boundary marking the growing region of vortical disturbances emanating from the corner of the cylinder. The debris boundary was initially axisymmetric and well-defined, but became more irregular and diffuse as time progressed (cf. Savaş 1987, figure 2).

\section{Modified flow: spin-down with rotating sidewall}

As already mentioned in the Introduction, whilst the endwall layers in a finite cylinder following impulsive spin-down to rest from a state of solid-body rotation 


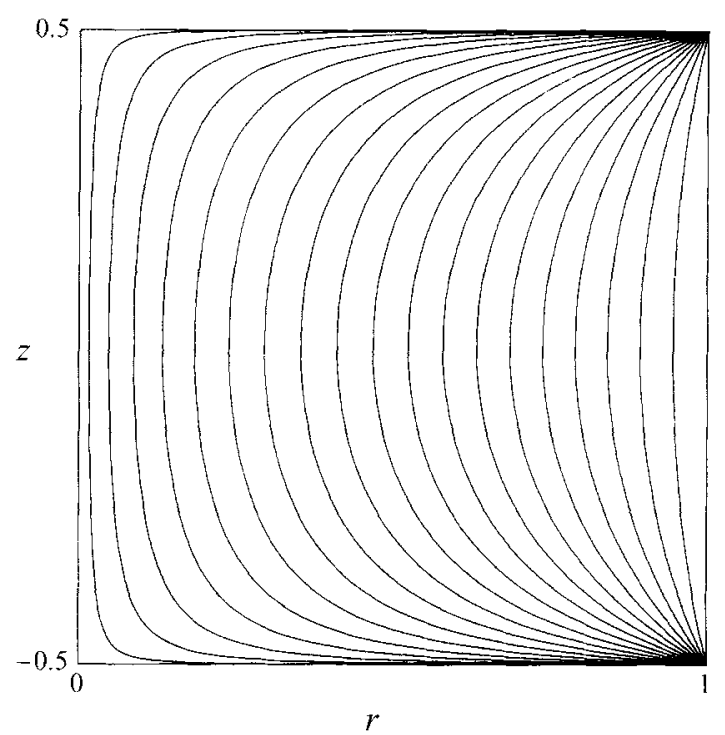

FIGURE 1. Contours of $\Gamma$ for Stokes flow and $H / R=1$. These are determined from the closed-form solution for the steady flow inside a cylinder with stationary endwalls and a rotating sidewall in the limit $R e \rightarrow 0$, given by Khalili \& Rath (1994).

are Bödewadt like, the flow has limitations for a study of the long-time stability of the endwall layers. As the flow spins down to rest, any transient instabilities are eventually stabilized. Also, the formation of corner vorticies and the susceptibility of the sidewall layer to $T-G$ instability, coupled with the radial inflow in the endwall layer, leads to ingestion of large-amplitude disturbances (corner vorticies and T-G cells) into the layer, causing major disruptions of the layer which are unrelated to the stability of the layer itself. In an attempt to alleviate these problems, a modified flow is also examined. The modified flow consists of a cylinder of aspect ratio $H / R$ with the fluid inside initially in solid-body rotation with the cylinder. The modification is that at $t=0$ only the endwalls are impulsively stopped and the sidewall continues to rotate.

The simple modification provides a number of improvements over the usual spindown flow for the study of the endwall layers. The modified flow has a non-trivial solution for all $R e$, whereas the spin-down flow evolves to rest (the trivial solution) for all $R e$. Even in the limit of Stokes flow $(R e=0)$, the modified flow has a non-trivial (and unique steady) solution. Khalili \& Rath (1994) provide an analytic solution of the modified flow at $R e=0$ (figure 1 is a plot of this flow for $H / R=1$ ).

The main feature of the modified flow, which exists for all $R e>0$, is that for all time following the impulsive stopping of the endwalls, all the vortex lines emanate from the corner $(r, z)=(1,-H / 2 R)$ and terminate at the corner $(r, z)=(1, H / 2 R)$. Further, these vortex lines are not parallel to the rotation axis; therefore, for $R e>0$, there is always an associated secondary flow with $\eta \neq 0$ and $\psi \neq 0$. Figure 2 is a plot of $v(r, z=0)$, the azimuthal velocity distribution at the cylinder half-height, for the $R e=0$ analytic solution of the modified flow. It shows that even at the centreline in the Stokes flow limit, the radial distribution of $v$ does not correspond to solid-body rotation. For $R e>0$, the meridional circulation, which is primarily directed in the outwards radial direction in the interior of the cylinder, also drives the flow away 


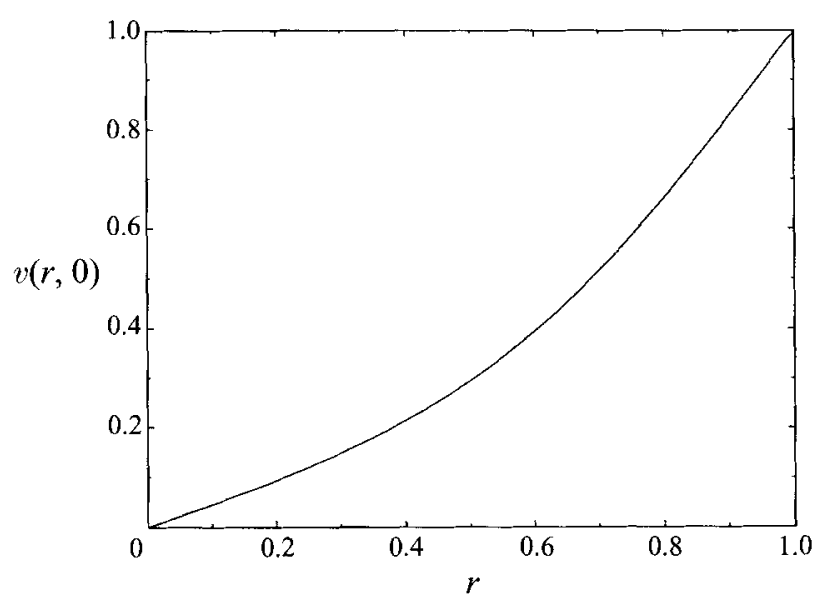

FIGURE 2. Radial variation of the azimuthal component of velocity $v$, at the cylinder half-height $z=0$ from the closed-form Stokes flow solution in figure 1.

from solid-body rotation. So, the modified flow is not ideal for the long-time study of the stability of Bödewadt flow, which requires steady solid-body rotation of the fluid above the boundary. However, in the following section we will see that for such a study it offers a vast improvement over the spin-down flow, which has $v(r, z)=0$ as $t \rightarrow \infty$, and for early times following the stopping of the endwalls, the modified flow is as good or better an approximation to a Bödewadt flow.

\section{Results}

The results are presented in the following order. Both the spin-down and modified flows are presented in tandem. First, comparisons with Savaş' experiments with $H / R=2$ and $R e=9632$ are presented. Then, the results of the present spindown experiment, with $R e=1.397 \times 10^{4}$ and $H / R=3.48$, are presented along with computations for the same conditions and the corresponding modified flow. Next, the development of the flow from $R e \approx 10^{3}$, where evidence of circular waves is just detectable, to $R e \approx 2 \times 10^{4}$ (spiral waves are reported by Savaş $(1983,1987)$ for $R e$ greater than about $3.8 \times 10^{4}$ ), are presented for $H / R=3.48$. Finally, the influence of $H / R$ is investigated for $R e=9632$.

It is instructive to view the flow evolution as a space-time plot of $\eta$, the azimuthal component of vorticity, computed on the top endwall and the top half of the sidewall ( $\eta$ on the other half of the sidewall and the bottom endwall is just a reflection of this due to the $Z_{2}$-symmetry). The horizontal axis on the space-time plots is time, from $t=0 \rightarrow 15$ and the vertical axis starts halfway up the sidewall at $(r=1, z=0)$, and progresses along the sidewall to the corner $(r=1, z=H / 2 R)$, then proceeds along the top endwall to the axis $(r=0, z=H / 2 R)$. The space-time plots show the 'footprints' on the walls of vortical waves in the various boundary layers. They also give a clear visual indication of the phase velocities of the waves. In order to help interpret these space-time plots, contour plots of $\eta$ in the top endwall boundary layer (covering the region $r=0 \rightarrow 1, z=H / 2 R-0.25 \rightarrow H / 2 R$ ) at various times are also provided. These clearly show the development of a multi-layered boundary layer structure and the evolution and propagation of the waves. A few plots of the contours of $\psi, \eta$ and $\Gamma$ over the half-meridional plane $r=0 \rightarrow 1, z=0 \rightarrow H / 2 R$ are also presented 
in order to put the space-time plots and the plots of $\eta$ in the top endwall boundary layer into perspective.

\subsection{Comparison with Savaş ( $R e=9632, H / R=2.0$ )}

The spin-down experiment of Savaş (1983) is first computed in order to reproduce the observed circular waves. The experiment was conducted in a cylinder of aspect ratio $H / R=2.0$ with $R=10.74 \mathrm{~cm}$. The initial rate of rotation was $\Omega=0.785 \mathrm{rad} \mathrm{s}^{-1}$ and the working fluid (water at $23{ }^{\circ} \mathrm{C}$ ) had a kinematic viscosity $v=0.0094 \mathrm{~cm}^{2} \mathrm{~s}^{-1}$, corresponding to $R e \approx 9632$. Figure 3 summarizes the evolution of the endwall boundary layer following an impulsive stop at $t=0$. The figure shows contours of $\eta$ in the neighbourhood of $z=H / 2 R$ for times $t=1 \rightarrow 10$. The left column (a) corresponds to the impulsive stopping of the whole cylinder (Savaş' experiment), while the right column $(b)$ corresponds to the modified flow where only the endwalls are impulsively stopped at $t=0$ and the sidewall continues to rotate. Figure 4 gives the corresponding space-time diagrams for the two flows for $0 \leqslant t \leqslant 15$.

At time $t=3$ (figure 5), the $\psi$ contours show that the flow near the endwalls is directed radially inwards, then radially outwards, then radially inwards, with increasing axial distance from the endwalls, leading to a multi-layered boundary layer structure. Note that the flow structure is virtually independent of whether the sidewall is stationary (figure $5 a$ ) or rotating (figure $5 b$ ), except for the $\Gamma$-contours (vortex lines) in the immediate vicinity of the sidewall. The $\psi$ - and $\eta$-contours only differ significantly between the two flows in the vicinity of the corner $(r=1$, $z=H / 2 R$ ). The endwall boundary layer is established in about one half-rotation time (i.e. by $t \approx 3$ ). This is somewhat sooner than for the boundary layer on a rotating disk following impulsive spin-up from rest. In that case, the layer takes about one rotation time to become established (Benton 1966). By $t=4$, spatial oscillations in the radial velocity have developed into recirculation cells which propagate along the axis to reach the midplane $z=H / 2 R$ by $t \approx 5$, as indicated by the closed contour in the space-time plot located near $t \approx 5$ and the cylinder half-height $z=0$ (figure 4). These are the 'solitary waves' and associated 'vortex breakdown bubbles' referred to by Valentine \& Miller (1994) when describing the spin-down flow for $R e=3000$ and $H / R=4$. Valentine \& Miller (1994) find that when these internal waves impinge on the midplane of the cylinder, the T-G instability of the sidewall layer is triggered. In the present calculations however, we find that the onset of $T-G$ cells is very much dependent on the level of spatial and temporal resolution used: the coarser the resolution, the earlier the onset time of the T-G instability. For sufficiently fine resolution $(n r=301, \delta t=0.01$ using second-order space and time integration), we find that the T-G instability does not set in until after two 'solitary waves' have impinged on the sidewall at the midplane (figure $4 a$ ); whereas with coarser resolution, we find that $T-G$ instability sets in following the first impingement. It is also interesting to note that even though the flow at these times has a large meridional recirculation zone in the interior, the vortex lines ( $\Gamma$-contours) show virtually no departure from being parallel to the axis. So, in the interior, the interaction between the primary flow $(\Gamma)$ and the secondary flow $(\psi)$ is quite minimal. This is in sharp contrast to situations where vortex breakdown occurs (e.g. Lopez 1990; Brown \& Lopez 1990; Lopez \& Perry 1992). In these cases, the vortex lines $(\Gamma)$ and the streamlines $(\psi)$ tend to coincide quite closely, leading to a large departure from cyclostrophic balance in the interior.

At $t \approx 2$, footprints of circular waves on the endwalls first appear (figures 3 and 4). Savaş (1983) reports that the waves become visible in his experiment after about $3 \mathrm{~s}$ 
(a)

$t=1$

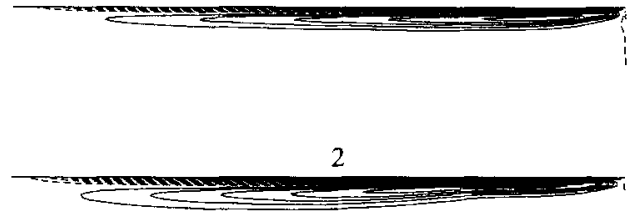

3

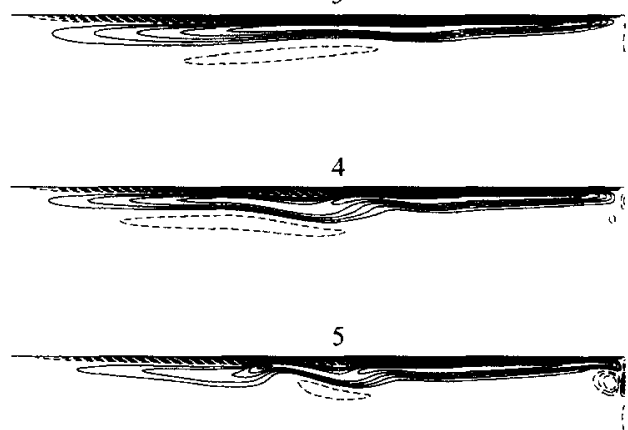

6

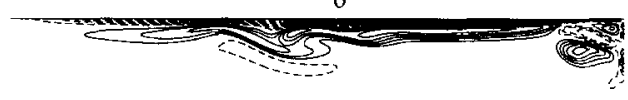

7

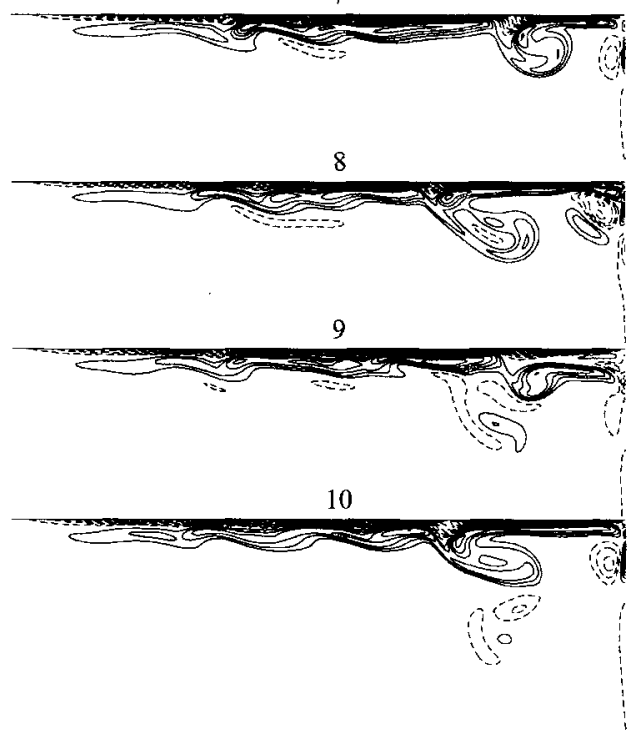

$t=1$

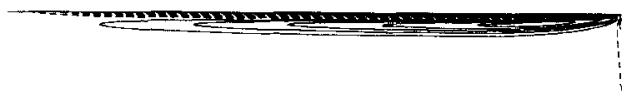

2

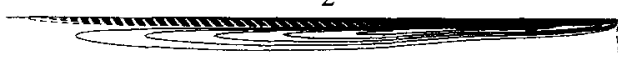

3

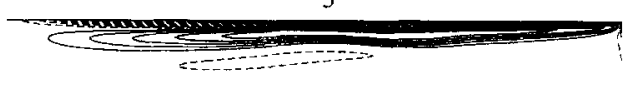

4

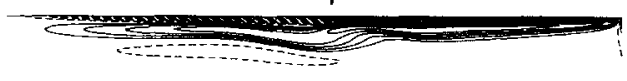

5

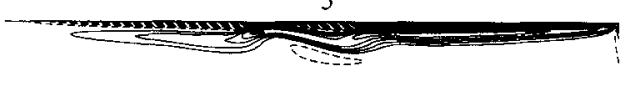

6

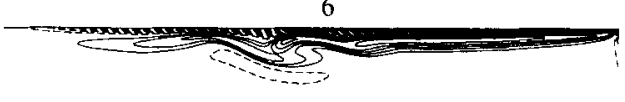

7

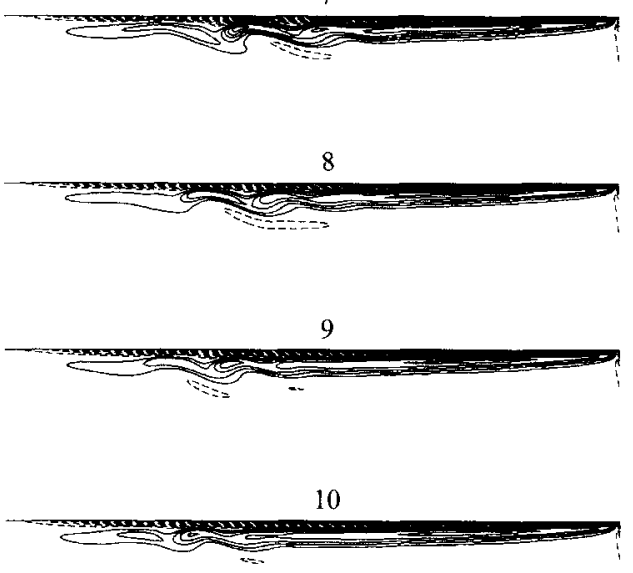

Figure 3. Contours of the azimuthal component of vorticity $\eta$, in the vicinity of the endwall $z=H / 2 R$, at various times as indicated, for $R e=9632$ and $H / R=2.0$. The contours in column (a) correspond to the spin-down flow where at $t=0$ all cylinder walls are impulsively stopped, and those in column $(b)$ correspond to the modified flow where only the endwalls are impulsively stopped, while the sidewall continues to rotate. The computations imposed $Z_{2}$-symmetry and used $n r=301, n z=301, \delta t=0.01$, and $a=0.1$. The solid lines are positive contours and the dashed lines are negative contours. 
(a)

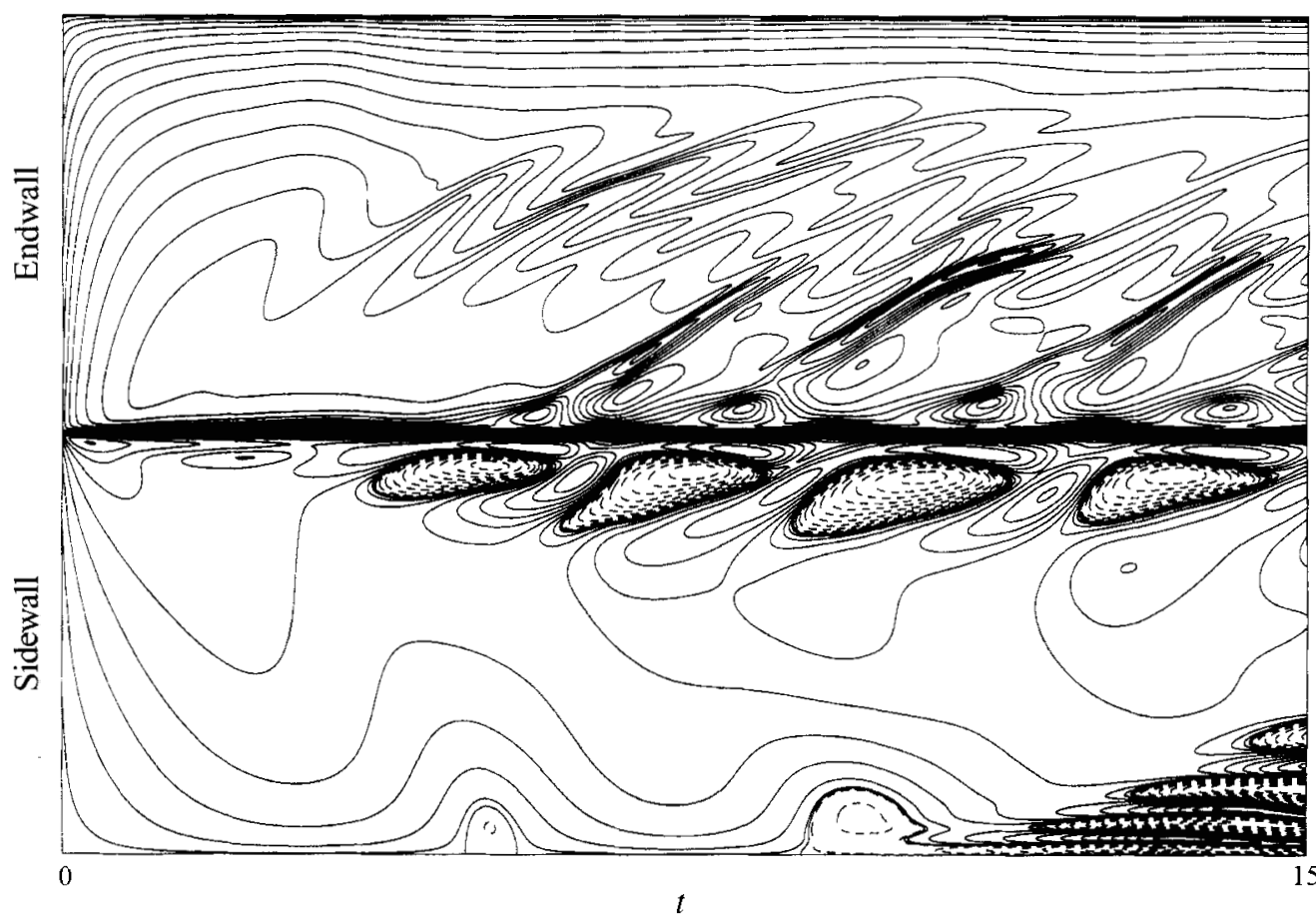

(b)

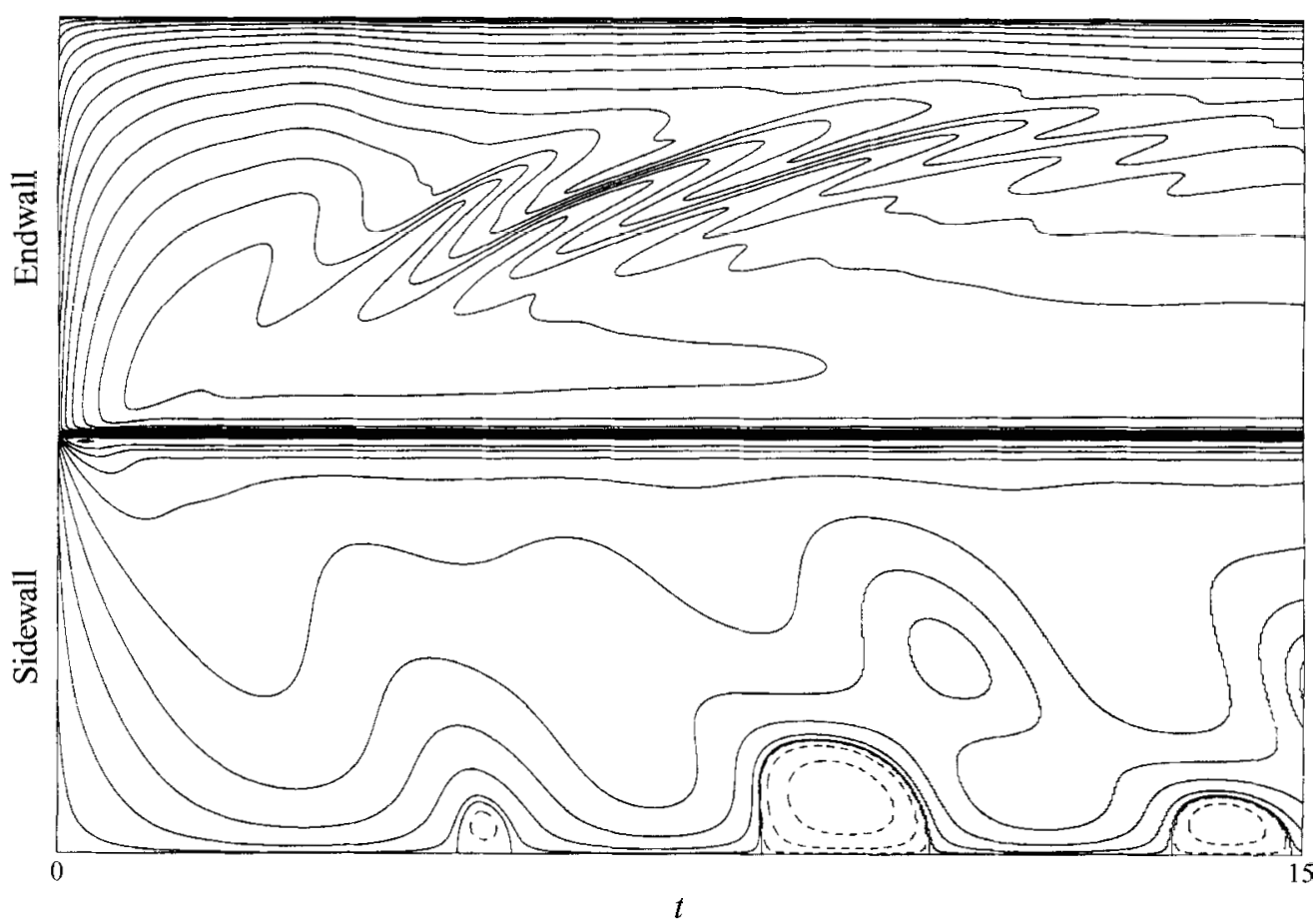

FiguRe 4. Space-time plots for $(a)$ the spin-down flow and $(b)$ the modified flow, as in figure 3 , consisting of contours of $\eta$ on the top endwall and the top half of the sidewall. Space is in the vertical direction, starting at the cylinder half-height $z=0$ on the bottom, progressing up to the corner $(r=1, z=H / 2 R)$, and then continuing along the endwall to the axis $r=0$ at the top of the plot. 

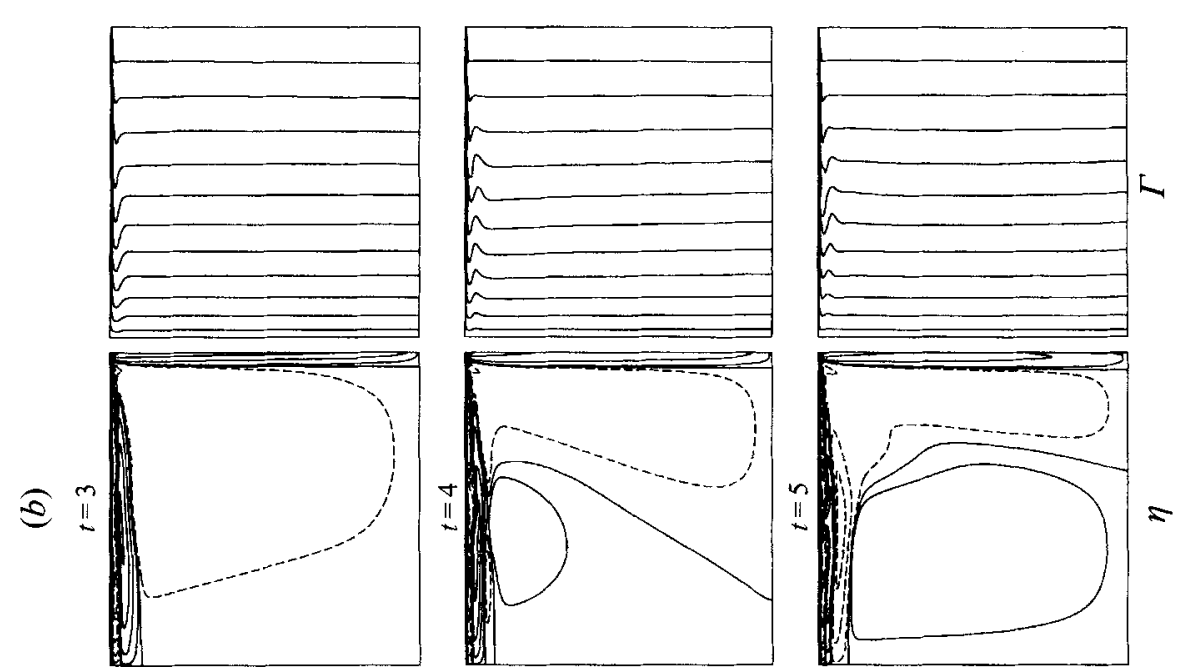

$\stackrel{9}{\Xi}$
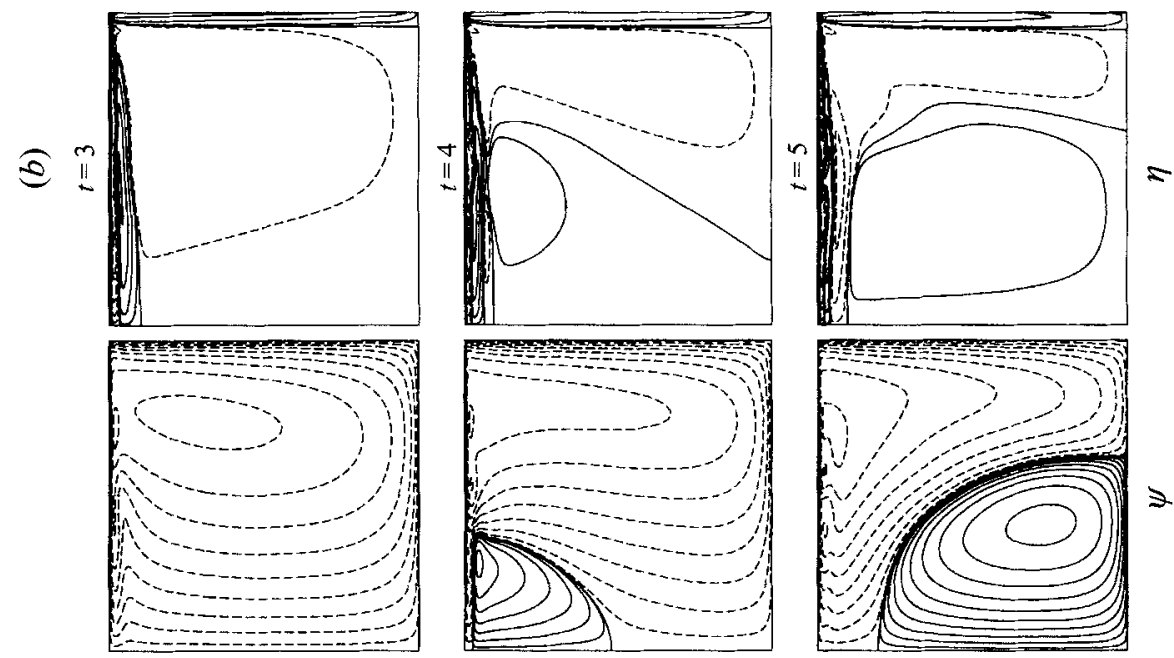

i

苛

프

ธิ

है

은

导

$-\quad 2$

2

?

음

言

$\rightarrow \quad \frac{1}{2}$

$\stackrel{9}{=}$

E
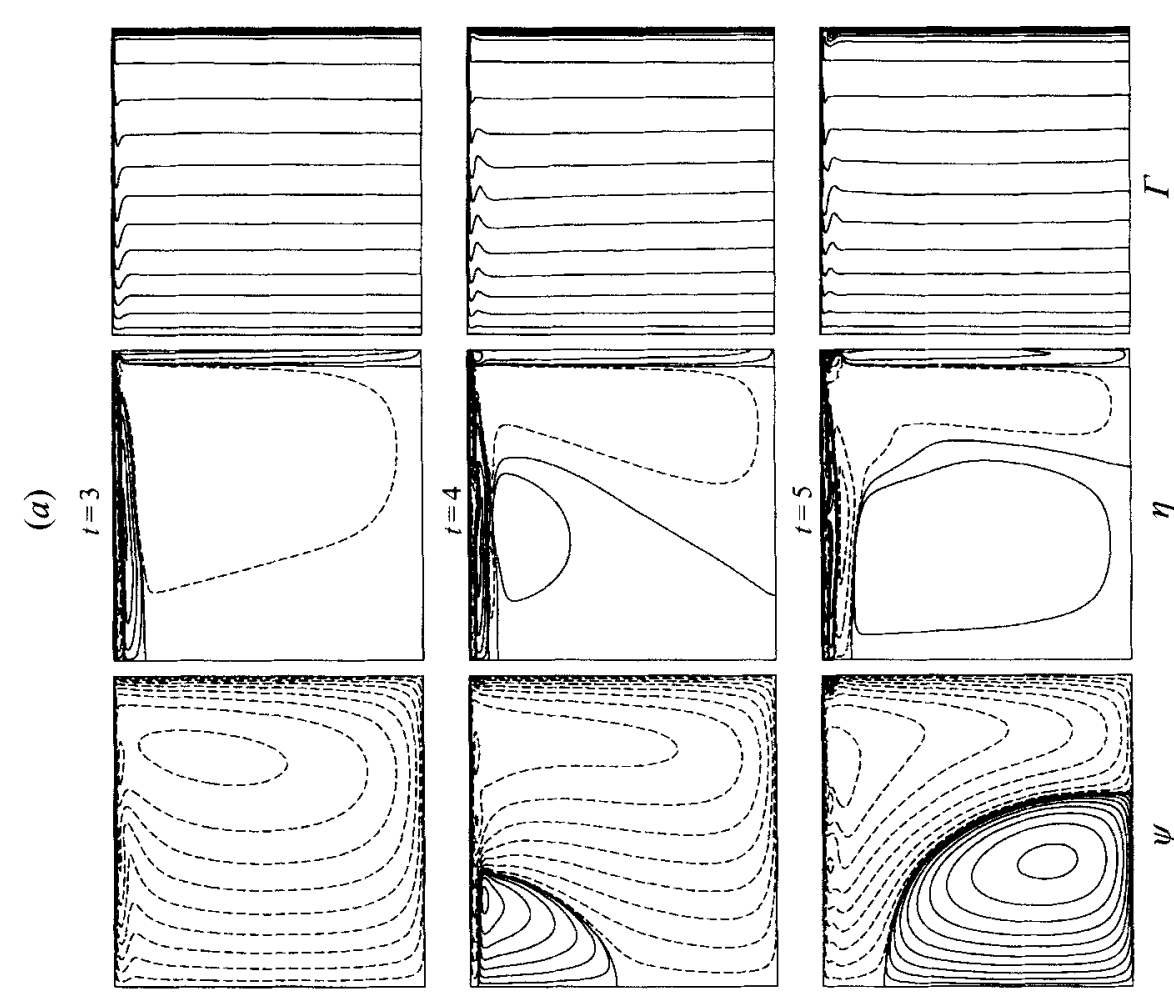

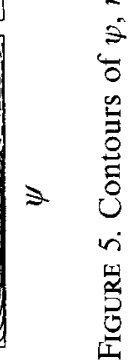


$(t \approx 2.36)$. These travel radially inwards on the endwalls with a gradual slowing until their footprints disappear at $r \approx 0.25$. The phase velocity of the first wave, estimated from figure $4(a)$ is $1.4 \mathrm{~cm} \mathrm{~s}^{-1}$ (dimensionalized by Savaş' (1983) experimental values of $R=10.74 \mathrm{~cm}$ and $\Omega=0.785 \mathrm{rad} \mathrm{s}^{-1}$ ). Savaş (1983) reports that the phase velocity estimated from his flow visualization is about $1.3 \mathrm{~cm} \mathrm{~s}^{-1}$ with a slight decrease towards the centre. The footprints of a second wave on each endwall become evident at $t \approx 4$ and these also travel radially inwards to $r \approx 0.25$, but more slowly $\left(\approx 0.9 \mathrm{~cm} \mathrm{~s}^{-1}\right)$ than the first waves. The slowing of the waves as they proceed radially inwards is also evident in the space-time plots (figure 4).

Defining a wavelength for the axisymmetric waves is not straightforward as both their spatial wavelength and their phase speeds are continuously changing as they progress radially inwards and as the secondary flow evolves (spins down). In his figure 3(e), Savaş (1983) provides a rather subjective method to define a wavelength for a particular wave at a particular point in its evolution. The range of his observed wavelength, non-dimensionalized by the cylinder radius, is $0.10-0.22$. From our computations presented in figure 3 , one could subjectively state that the wavelengths are also in the range $0.1-0.2$.

Savaş $(1983,1987)$ observes that the endwall boundary layer is stable near the origin, as evidenced by the relative calmness of the flow in the central part of the disk in all the flow-visualization observations. He defined a local Reynolds number, $r(\Omega / v)^{0.5}$, and observed that for values less than some critical value, the waves were not detected. From his various measurements, he concluded that the critical value is $25 \pm 5$. The present numerical results agree with those observations. Evidence of the waves not progressing all the way to the origin is clear in both figures 3 and 4 . Further, the two figures indicate that the waves do not proceed towards the origin beyond $r \approx 0.28$ for the $R e=9632, H / R=2.0$ case, giving a critical local Reynolds number of $\approx 27.5$.

At time $t \approx 4$, there is evidence (figures $3 a$ and $4 a$ ) of a corner vortex forming and by $t=5$ this vortex is very distinct. At these times (figures $5 a$ ), the sidewall layer shows no hint of any growth of disturbances due to instability. At $t \approx 5$, the corner vortex is ingested into the endwall layer. Initially, it travels with a uniform speed until it reaches $r \approx 0.67$, then its footprint appears much the same as those of the earlier endwall waves. Savaş' (1983) figure $2(d)$, corresponding to $t=6.6$, shows the presence of the debris boundary of vortical disturbances emanating from the corner to be located at $r \approx 0.75$. At $t=6$, figure $3(a)$ shows the $\eta$-front of the ingested corner vortex at $r \approx 0.89$ and at $t=7$ it is located at $r \approx 0.78$, placing it at $r \approx 0.82$ at $t=6.6$. Also, from the space-time plot (figure 4), the footprint of the ingested corner vortex on the endwall at $t=6.6$ is centred at $r \approx 0.81$. Following the ingestion of the first corner vortex, another forms and is subsequently ingested at $t \approx 7.5$.

Beyond $t \approx 6$, the flow evolution in the endwall boundary layer in the spin-down flow is dominated by the ingested corner vortices. There is a continual ingestion of these vortices into the endwall layer and any further study of the Bödewadt-type flow is severely compromised. However, comparing the spin-down flow and the modified flow with the rotating sidewall for $r<1-\delta$, where $\delta$ is approximately the sidewall layer thickness at a particular point in time, the two flows are virtually indistinguishable up to the time when the corner vortices are ingested in the spindown flow. Note that corner vorticies never form in the modified flow. Even beyond the time when the corner vortices are ingested in the spin-down flow, the interior of the two flows agree to a large degree. In particular, the internal waves reported by Valentine \& Miller (1994) for the spin-down flow are also present in the modified flow. 
Further, the structure of the axisymmetric waves in the endwall boundary layer is the same in the two flows, up until the upstream influence of the ingested corner vortex becomes significant at $t \approx 8$ (figure 3 ). This correspondence between the two flows indicates that the axisymmetric waves reported by Savaş $(1983,1987)$ are not due to any centrifugal instability of the sidewall layer in his experiment, nor to vortical disturbances ingested from the corner. It provides evidence that these waves are a local response to the underlying structure of the endwall boundary layer; in essence, a form of instability of the Bödewadt flow, as indicated by Savaş $(1983,1987)$.

\subsection{Comparison between experiment and numerics for $R e=1.397 \times 10^{4}$ and $H / R=3.48$}

The measurements of the radius of the bright rings corresponding to the circular waves from the experiment described in $\$ 4$ are presented on a space-time plot in figure 6. Figure $6(a)$ shows the experimental data superposed onto a space-time plot of contours of constant azimuthal vorticity, $\eta$, from the numerical computations to aid in comparisons. Figure $6(b)$ is a detail of the trajectories over the period $3.00 \leqslant t \leqslant 8.25$ and $0.20 \leqslant r \leqslant 0.75$. In this experiment, five circular wave disturbances were identified before the debris from the edge dominated the boundary layer flow. The first observable waves, labelled 1 and 2 , are spatially broad disturbances that clearly propagate at nearly equal and constant dimensionless speed $\Delta r / \Delta t=-0.136$. The remnants of these disturbances, especially that of wave 2 , seem to be fossilized in the fluid at small radii. Waves 3,4 , and 5 , on the other hand are much narrower and actively follow the back side of local folds in the $\eta$-contours. This trend is especially evident for wave 4 which starts out on the back side of a single vorticity fold, but splits into two disturbances $4 a$ and $4 b$ prior to the development of a new local vorticity fold develops. Note that the speed of waves 3,4 , and 5 decreases with time, as do the corresponding waves in the numerics, in contrast to the constant propagation speed of the first two observable waves in the experiment.

The observed debris boundary, indicated in figure 6 by the open circles, tracks the progression of the ingested corner vortex from the numerics very well. This is especially so during the early evolution of the corner vortex until it reaches $r \approx 0.5$.

The wave trajectories clearly follow the trend in the vorticity fold pattern computed for the experimental conditions. Moreover, it is apparent that the measured debris boundary, first detected at $t \approx 5.4$ and $r \approx 0.88$, was triggered by the first corner vortex that appeared at $t \approx 5.0$ in the computation. We conclude that the numerical simulation corroborates the experimental observations both qualitatively and quantitatively.

The numerical development of the endwall layer can also be followed in figure 7 where, as in figure 3 , the $\eta$-contours in the vicinity of the endwall at various times as indicated are plotted for both $(a)$ the spin-down flow (corresponding to the experiment), and $(b)$ the modified flow. The bifurcation of one wave into two, as observed in the experiment for wave 4 splitting into waves $4 a$ and $4 b$, can be seen in figure 7 between $t=7$ and $t=8$. This splitting of the wave is evident in both the spin-down flow and the modified flow. It is interesting to note that even at $t=10$, by which time a number of corner vortices have been ingested into the layer and have propagated radially inwards more than one third of the way to the origin, the boundary layer structure for the two flows, for $r<0.3$, remains remarkably similar.

The agreement in the wave structure between the spin-down flow and the modified flow, together with the advantages of the modified flow, clearly indicate it to be a suitable flow in which to study the axisymmetric endwall waves. Further, the close 
(a)
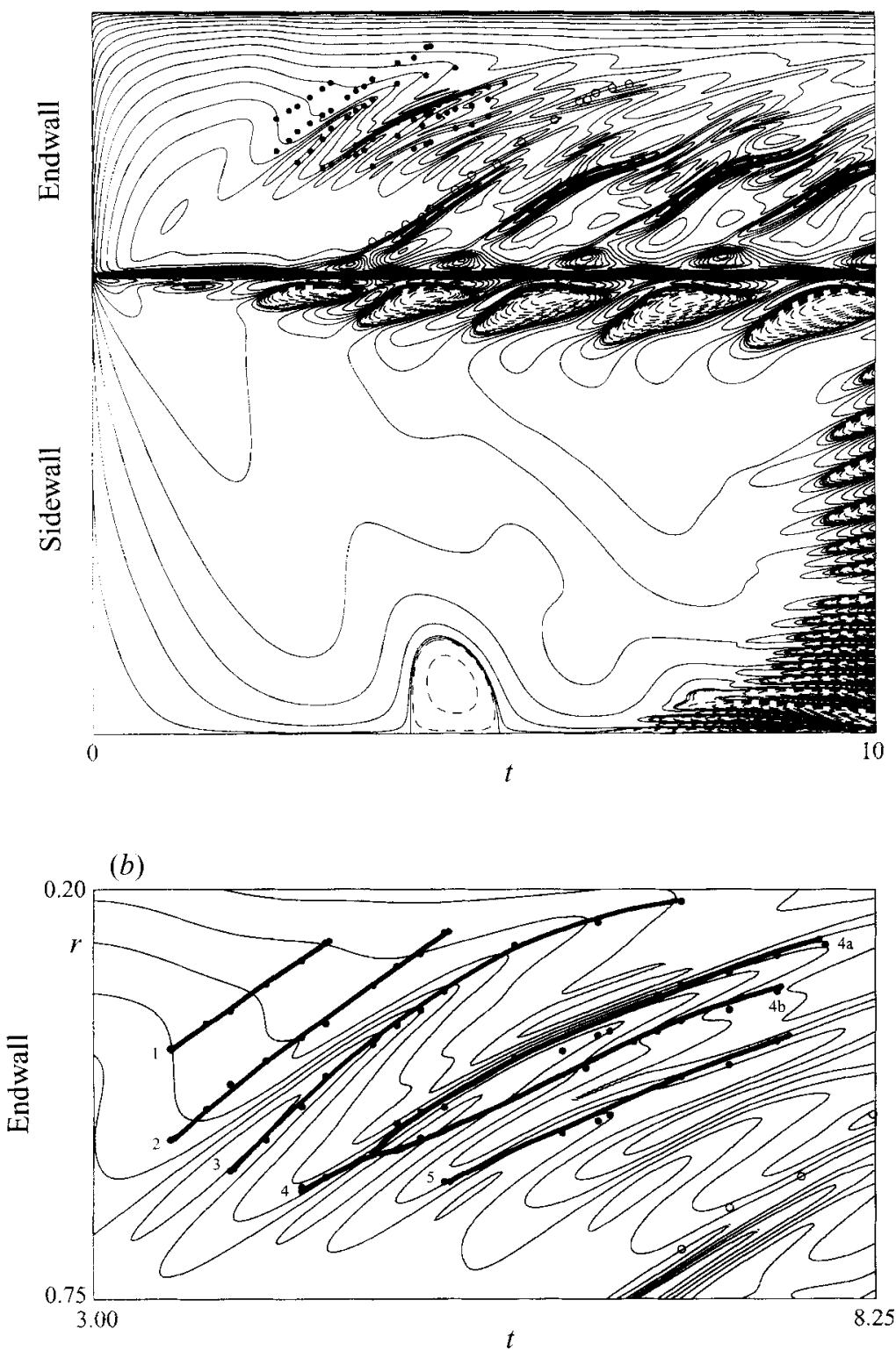

FIGURE 6. Space time plots for the spin-down flow with $R e=1.397 \times 10^{4}$ and $H / R=3.48$. (a) Locations of the axisymmetric waves in the endwall boundary layer, $\bullet$, and the debris boundary, $\circ$, from the experimental measurement, together with contours of $\eta$ on the top endwall and the top half of the sidewall. $(b)$ A detail of $(a)$ over $3.00 \leqslant t \leqslant 8.25$ and $0.20 \leqslant r \leqslant 0.75$. The computation imposed $Z_{2}$-symmetry and used $n r=301, n z=523, \delta t=0.01$, and $a=0.1$. The solid lines are positive contours and the dashed lines are negative contours.

agreement between the numerics and the present experiment and those of Savaş (1983, 1987) indicates that the numerical simulations are a suitable tool for the investigation of these waves. In the following subsections, the influence of the two governing parameters, $R e$ and $H / R$, on the waves is studied numerically in the modified flow. 
(a)
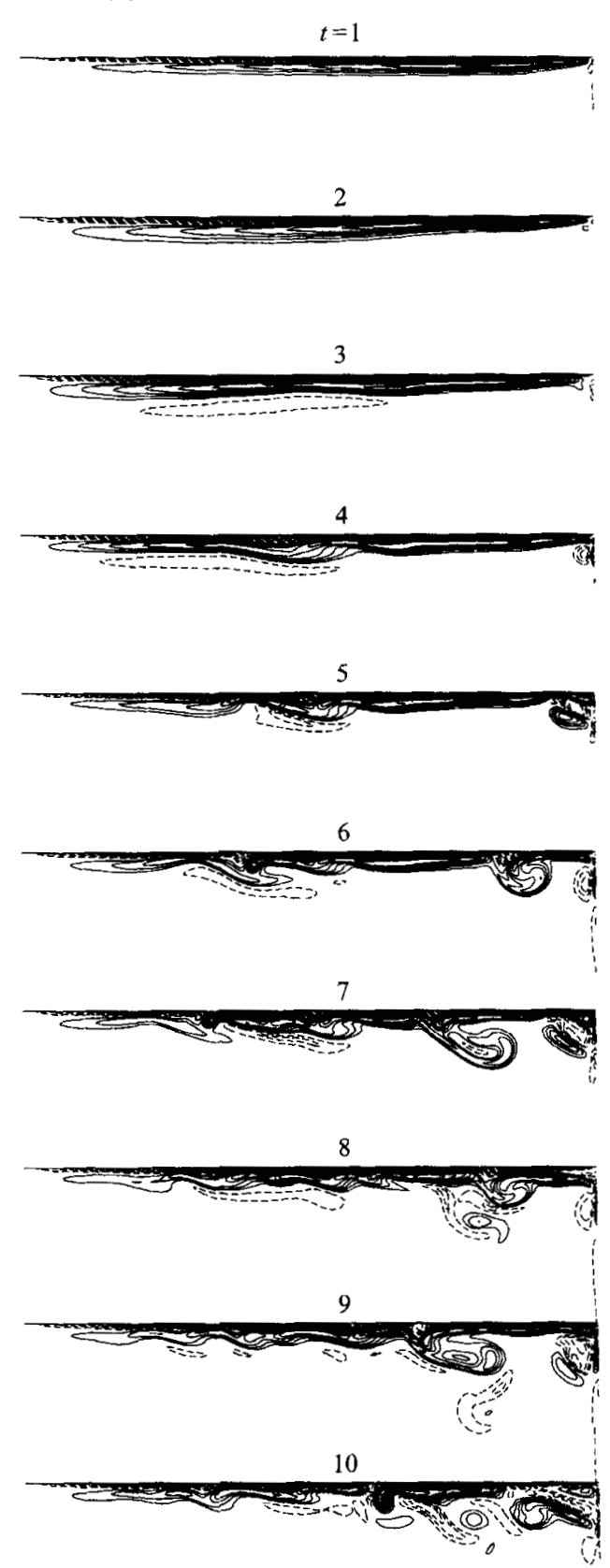

(b)

$$
t=1
$$

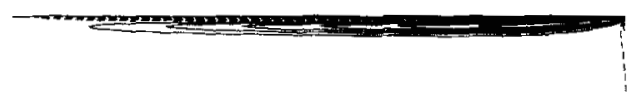

2

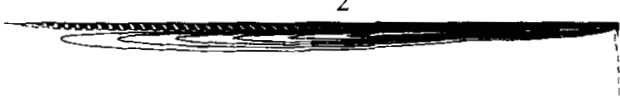

3
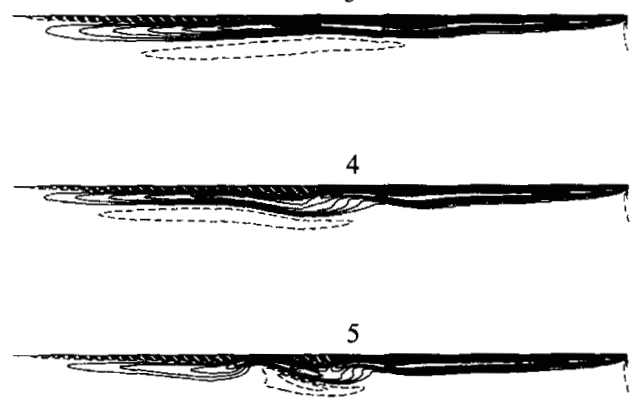

6

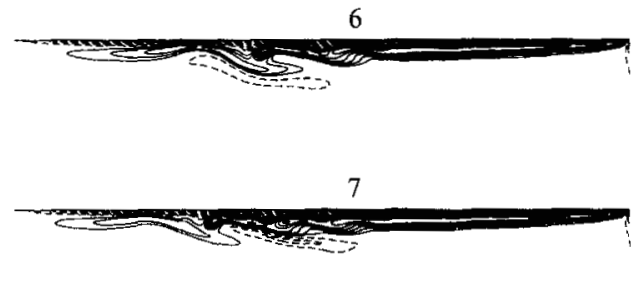

8

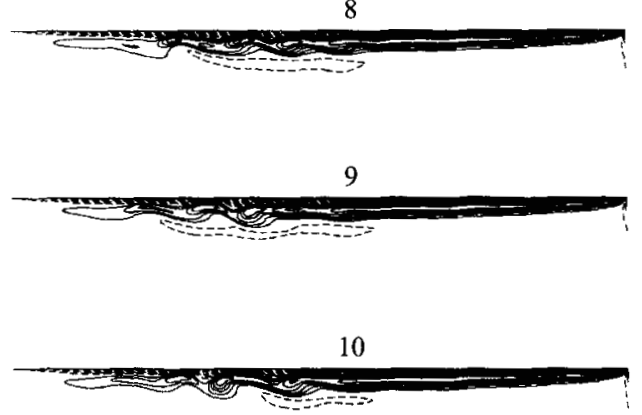

FIGURE 7. Same as in figure 3, but for $R e=1.397 \times 10^{4}, H / R=3.48$, and $n z=523$. 
6.3. Flow development with increasing Re for $H / R=3.48$ for the modified flow

Having established that the axisymmetric waves on the endwall boundary layers are a response to the local structure of the boundary layers, we now investigate the waves in the modified flow where the sidewall continues to rotate, thus eliminating the presence of corner vorticies and centrifugal instability of the sidewall layer.

The development of the endwall boundary layer as $R e$ is increased is summarized in a sequence of space-time plots (figure 8) for $H / R=3.48$. From these, it is clear that even for $R e$ as low as $1.25 \times 10^{3}$, a pair of undulations develops following the impulsive stop. Both these undulations are spatially broad and of relatively smallamplitude. One would expect that such small-amplitude, broad waves would not be detectable in a flow visualization experiment using aluminium flakes or Kalliroscope. By $R e=2.5 \times 10^{3}$, the wave amplitude has increased, the wavelength has decreased, and the phase speed has increased significantly. The spin-down and the modified flows are virtually indistinguishable for a considerable time, and for $t>5$, the main contributing factor to their distinction is that the spin-down flow is evolving to rest, i.e. there is no applied torque in that flow, whereas in the modified flow there is still torque applied at the sidewall. For low $\operatorname{Re} \leqslant 2.5 \times 10^{3}$ and $t \leqslant 10$ there is not even the presence of a corner vortex in the spin-down flow to distinguish the two flows. Corner vortices in the spin-down flow with $H / R=3.48$ appear at $t \approx 5$ for $R e=5 \times 10^{3}$ and earlier for larger $R e$.

As $R e$ is increased through $5 \times 10^{3}, 9.632 \times 10^{3}, 1.397 \times 10^{4}$, and $1.74 \times 10^{4}$, there is a clear trend that the waves travel faster with larger $R e$, and their wavelength is smaller with larger $R e$. For $R e \leqslant 9.632 \times 10^{3}$, it is apparent from figure 8 that each wave travels radially inwards with no noticeable interaction with preceding or following waves, that they remain a single entity, and fade away as they reach $r \approx 0.2$. For larger $R e$, however, we find that waves bifurcate, as noted in the experiment ( $\$ 6.2)$, and coalesce. The coalescence events occur as a wave slows down as it proceeds radially inwards; it is slowed further still by the presence of a preceding wave, and a following wave 'runs into it', coalescing with it. These events are discernible in the $R e=1.74 \times 10^{4}$ case in figure 8 , where at later times, at about $r \approx 0.5$, two folds become one. It is not as easy to see these events in the $\eta$-contours of figure 9 , as the 'snap-shots' are given too far apart. However, it is particularly evident from these types of plots when they are animated with fine temporal resolution on a graphics workstation.

For all the cases of the modified flow considered, when they were evolved to longer times $(t>60)$, it was found that the waves eventually disappeared and the boundary layer had little radial variation (apart from that due to its finite length), although the Bödewadt-type spatial oscillations in the axial direction were still present.

We find two trends from the various computations of the modified flow. As time progresses, the induced secondary meridional flow, which in the interior primarily consists of a radial flow towards the sidewall, advects the vortex lines to larger radius. The vortex lines remain essentially parallel to the axis. So, although the interior continues to rotate with $\Gamma$ largely independent of $z$, the flow is far removed from a state of solid-body rotation. The other trend is that with smaller $R e$, the vortex lines migrate out towards the sidewall faster and the asymptotic long-time limit of the flow is further from solid-body rotation. Figure 2 shows the $v$-profile at the cylinder half-height, illustrating the departure from solid-body rotation $(v=r)$ in the limit as $R e \rightarrow 0$, at steady state. The end result of this departure is that we no longer have a situation corresponding to the Bödewadt flow. In essence, the unstable endwall 

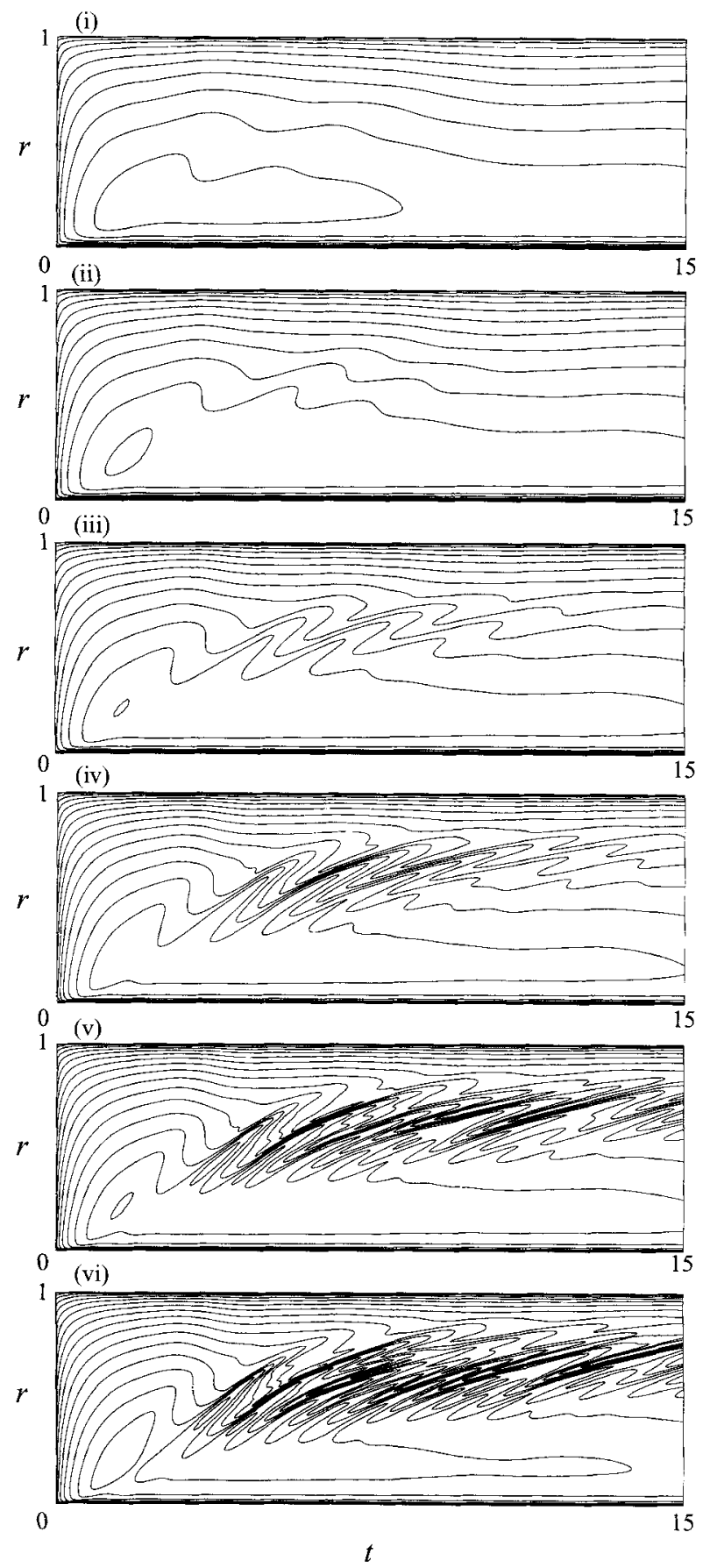

FIGURE 8. Space-time plots for the modified flow with $H / R=3.48$ and $R e$ as indicated. In all cases $n r=301, n z=523, a=0.1$, but $\delta t$ varies for $\operatorname{Re}$ as follows: (i) $\operatorname{Re}=1.25 \times 10^{3}, \delta t=1.25 \times 10^{-3}$, (ii) $R e=2.5 \times 10^{3}, \delta t=2.5 \times 10^{-3}$, (iii) $R e=5 \times 10^{3}, \delta t=5 \times 10^{-3}$, (iv) $R e=9.632 \times 10^{3}, \delta t=10^{-2}$, (v) $R e=1.397 \times 10^{4}, \delta t=10^{-2}$, (vi) $R e=1.74 \times 10^{4}, \delta t=10^{-2}$. 
(a)

$t=1$

2
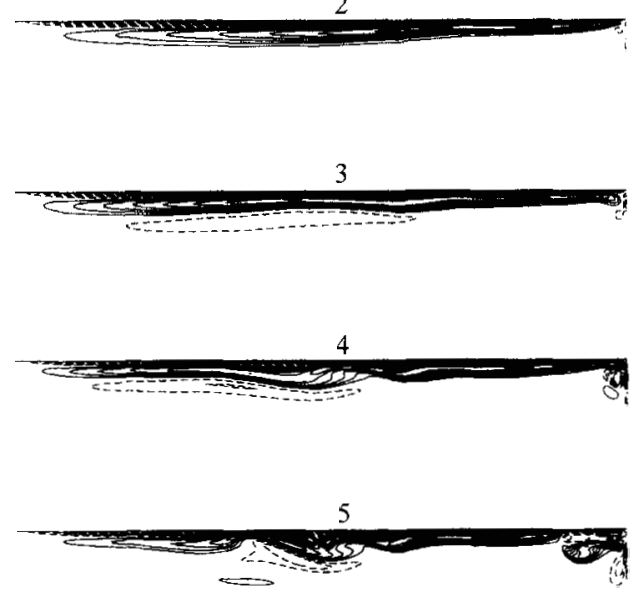

6
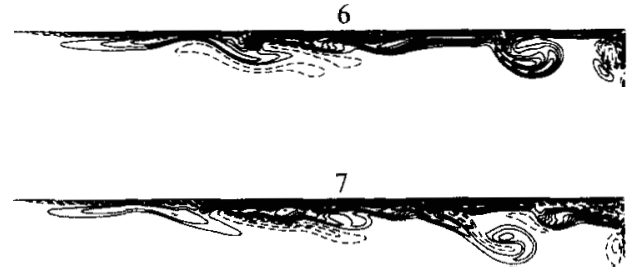

8

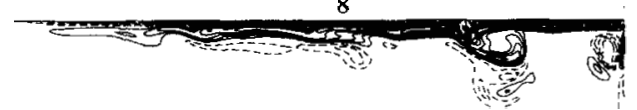

9

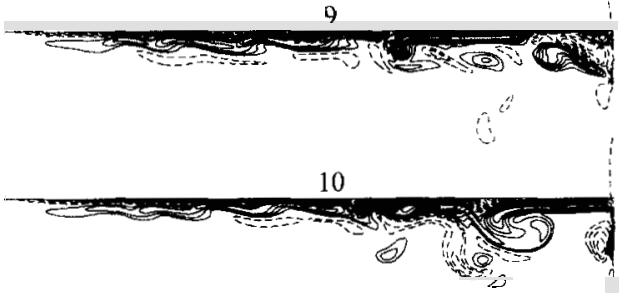

(b)

$t=1$

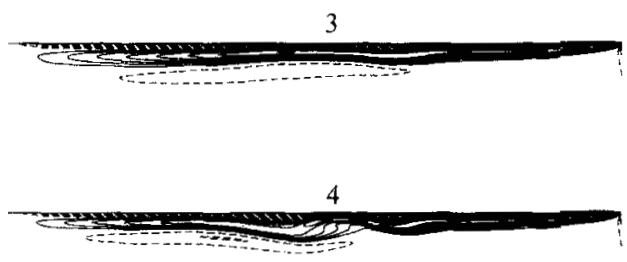

5

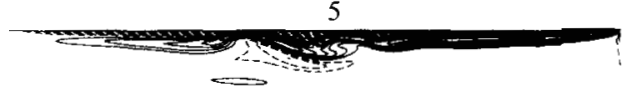

6

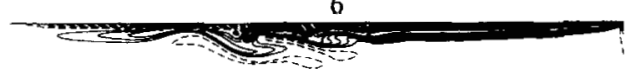

7

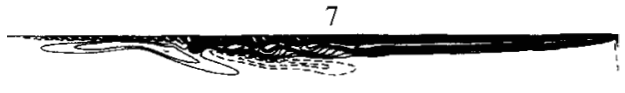

8

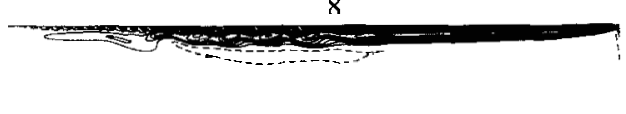

9

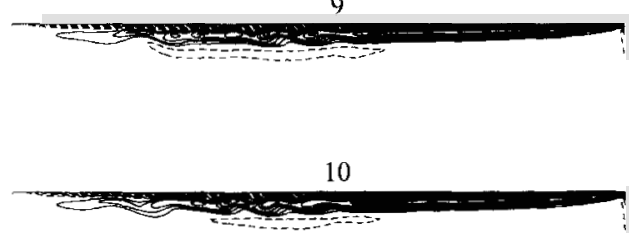

FIGURE 9. Same as in figure 3 , but for $R e=1.74 \times 10^{4}, H / R=3.48$, and $n z=523$. 


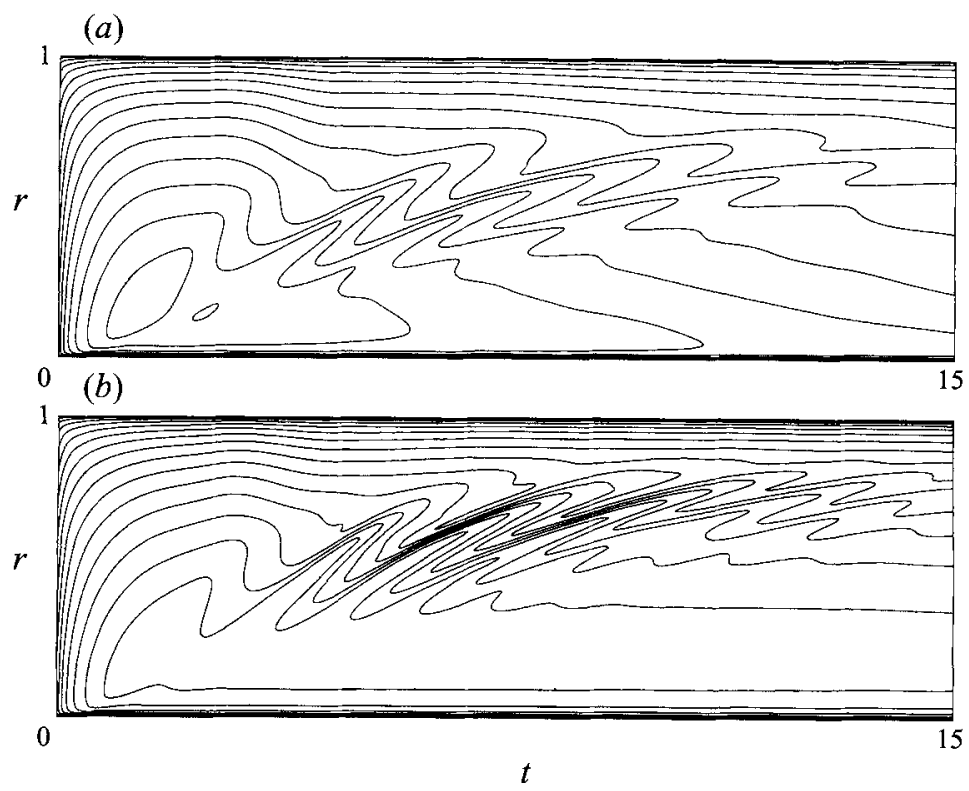

Figure 10. Space-time plots for the modified flow with $R e=9.632 \times 10^{3}$. (a) $H / R=0.5, n r=301$, $n z=76, a=0.1$, and $\delta t=10^{-2}$. (b) $H / R=8.0, n r=301, n z=1201, a=0.1$, and $\delta t=10^{-2}$.

boundary layer stabilizes itself by inducing a secondary meridional circulation which advects the vortex lines responsible for the layer's instability into a configuration which no longer results in an unstable endwall layer.

\subsection{Influence of $H / R$ for $R e=9632$ for the modified flow}

Here we present sample results for $R e=9632$ and $H / R=0.5,2.0$, and 8.0 in the modified flow. The main result here is that the waves are spatially broader, travel more slowly, and have smaller amplitude as $H / R$ is reduced. We have found that as $H / R$ is increased beyond $\approx 5$, its effects rapidly diminish, and that $H / R=8.0$ is large enough for the flow to be considered at an asymptotic limit of large $H / R$. We did not explore the limit $H / R \rightarrow 0$. Figure 10 together with figure $4(b)$ show the spacetime plots for the three $H / R$ values, demonstrating this trend. The corresponding $\eta$-contours in the endwall region are displayed in figures 11,3 , and 12 , both for the spin-down flow and the modified flow. These figures also show the above trend.

The diminished wave activity for smaller $H / R$ at fixed $R e$ can be understood as follows. For small $H / R$, the meridional flow is mostly radial: outwards in the interior and inwards in the layer. The outwards radial flow in the interior tends to advect the vortex lines to larger radii, which still remain essentially parallel to the axis (Taylor-Proudman theorem). In so doing, the interior flow is no longer in solid-body rotation and the corresponding boundary layers on the endwalls, while still showing the characteristic oscillations of the Bödewadt solution, have spatial oscillations in the axial direction of smaller amplitude. This adjustment of the primary flow (the advection of the vortex lines to larger radii) by the secondary flow acts to stabilize the boundary layer. In contrast, for $H / R$ large, the meridional flow is mostly axial, away from the endwall layers in the interior and in towards the endwalls in the sidewall layer with a weak radial flow from the interior into the 
(a)

$t=1$

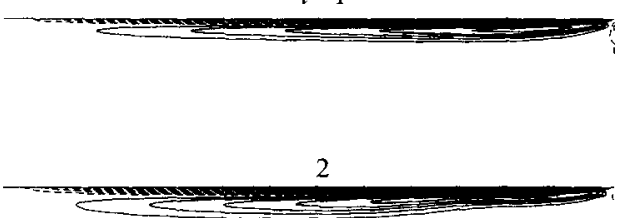

3

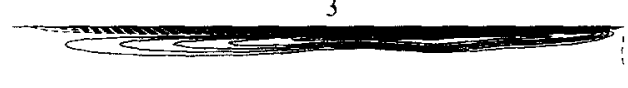

4
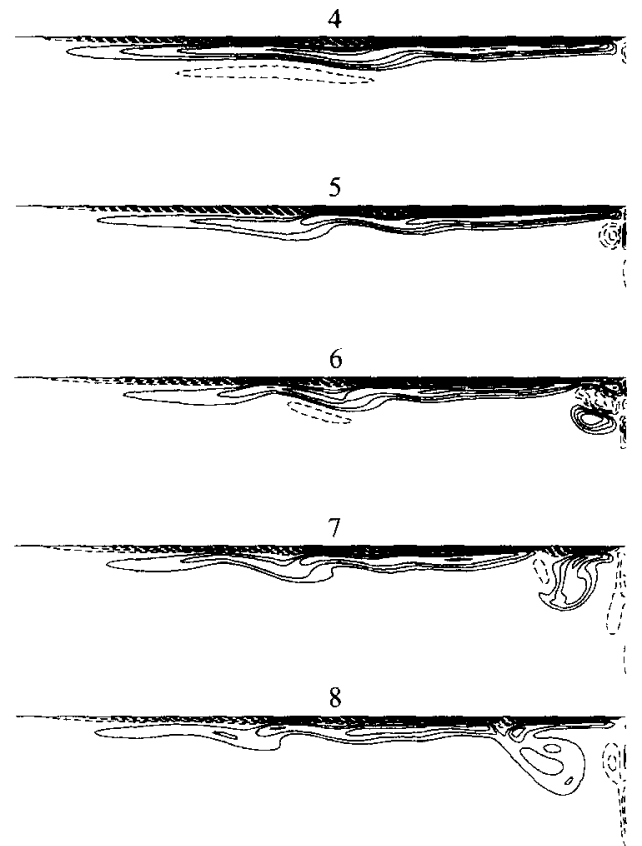

9

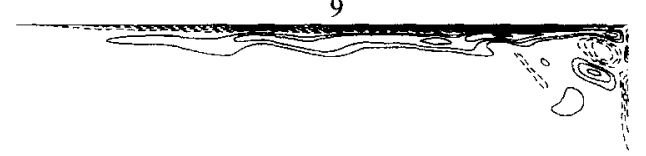

10

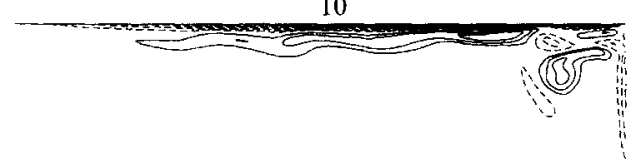

(b)
2

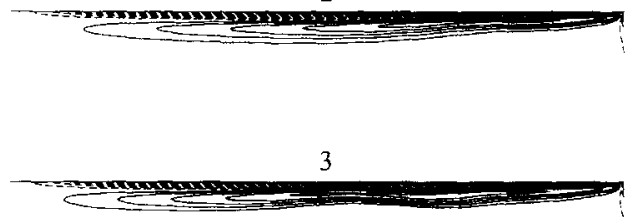

4

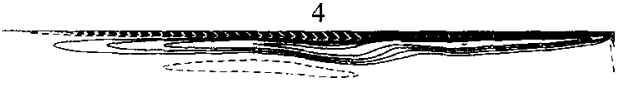

5

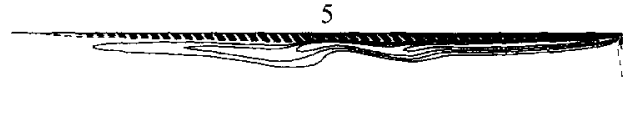

6

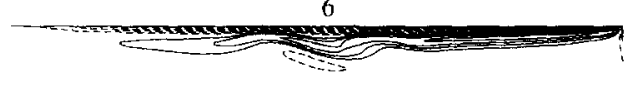

7

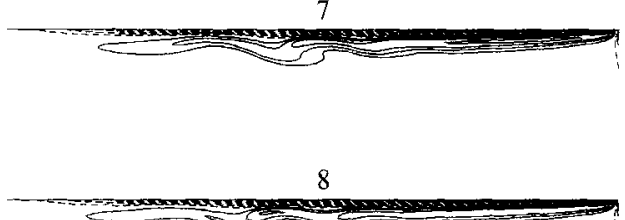

9

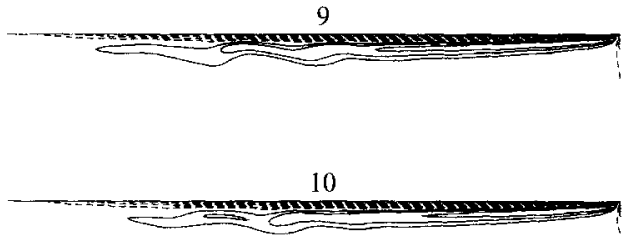

FIGURE 11. Same as in figure 3, but for $H / R=0.5$ and $n z=76$.

sidewall layer near $z=H / 2$, which for large $H / R$ is far removed from the endwalls. The relatively weaker radial flow in the interior, compared with small- $H / R$ cases, does not advect the vortex lines to such large radii. In comparison, the interior flow is closer to solid-body rotation and the spatial oscillations in the endwall layer are more pronounced. 
(a)

$t=1$

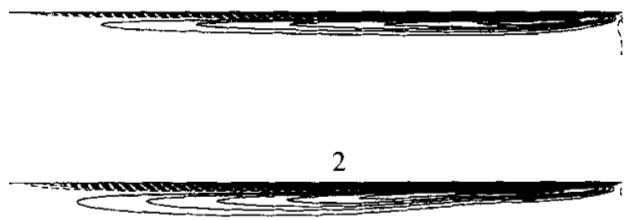

3
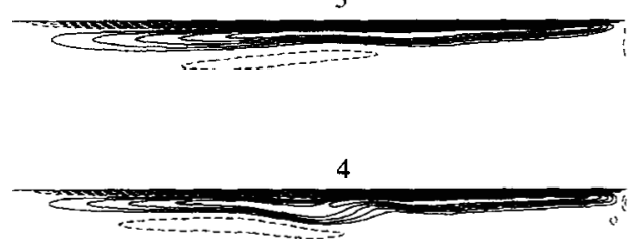

5

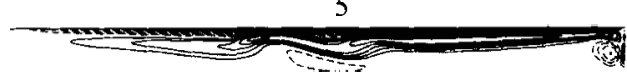

6

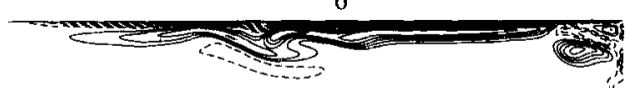

7

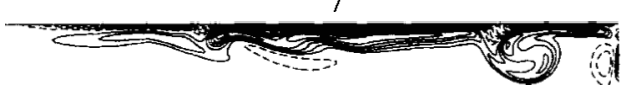

8

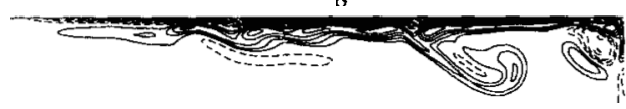

9

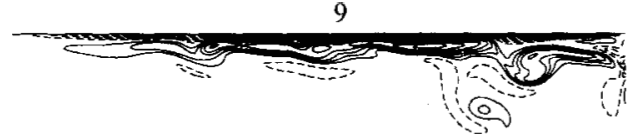

10

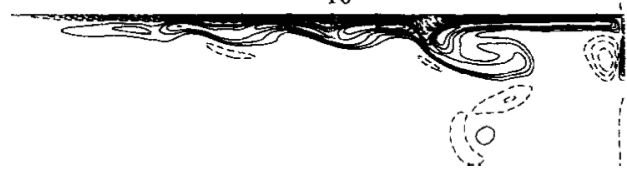

$t=1$

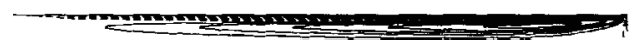

2

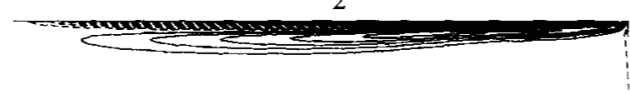

3
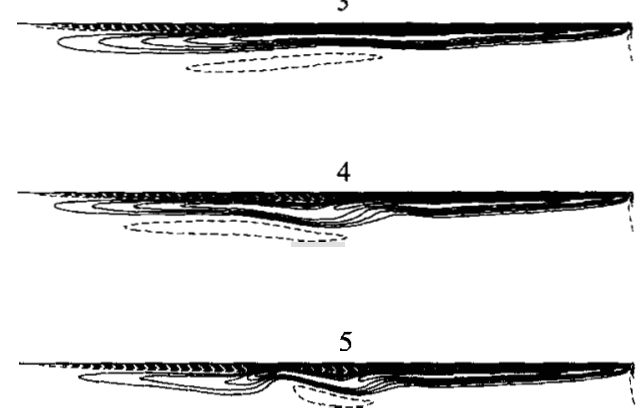

6

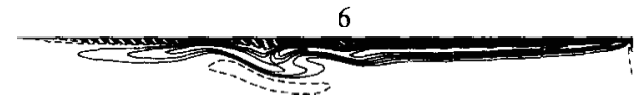

7

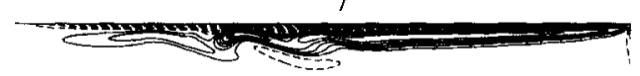

8

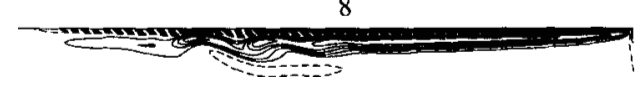

9

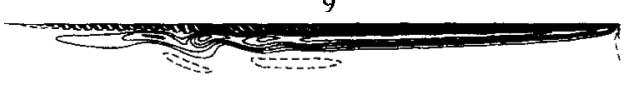

10

FIGURE 12. Same as in figure 3 , but for $H / R=8.0$ and $n z=1201$. 


\section{Conclusions}

The circular waves observed by Savaş $(1983,1985)$ on the endwall boundary layers in a cylinder with the fluid initially in solid-body rotation, following an impulsive stop, are an instability of the endwall layers. The form of these waves is dependent on the local structure of the boundary layer, and does not depend on the nature of the flow at the edge of the disk endwalls. Since the structure of the endwall boundary layers immediately following the impulsive stopping of the cylinder is of Bödewadt type, these waves are seen to provide a breakdown of the self-similar structure of the boundary layers. The long-time development of the waves cannot be followed in the spin-down flows as corner vortices formed at the edge of the endwall, and Taylor-Görtler vorticies as a result of the centrifugal instability of the sidewall layer, are ingested into the endwall layer early in the flow evolution. A modified flow where only the endwalls are impulsively stopped has essentially the same initial endwall boundary layer structure. In this flow, the evolution of the waves can be followed for long times. However, owing to the interaction between the primary and secondary flow, the vortex lines are swept out to larger radii, so there can be no solid-body rotation in the interior and there is less sharp bending of the vortex lines in the endwall boundary layer, resulting in a restabilization of the layer and the disappearance of the circular waves. Computations up to $R e \approx 2 \times 10^{4}$ have been performed and the circular waves in all cases eventually disappeared. On the bases of the computations presented here, it is concluded that the Bödewadt layer in the modified flow is unstable, but the shear across the Bödewadt layer attenuates as the interior flow adjusts to the secondary meridional flow, leading to a restabilization of the layer. The results suggest however, that for large $H / R$ and large $R e$ the interior will remain closer to solid-body rotation and in that case the circular waves will persist for a longer period of time. They must eventually die out in the classic spindown problem as the flow evolves to rest, but perhaps they will persist indefinitely in the modified flow with the rotating sidewall.

The authors wish to thank John Hart and Scott Kittleman for use of their rotating tank facility which was used to make preliminary visualizations of these spin-down flows prior to the measurements reported in this paper. The work was partly supported by NSF grant DMS-9512483.

\section{REFERENCES}

Benton, E. R. 1966 On the flow due to a rotating disk. J. Fluid Mech. 4, 781-800.

BödEwadT, U. T. 1940 Die Drehströmung über festem Grunde. Z. Angew. Math. Mech. 20, 241-245. BRAGG, S. L. \& Hawthorne, W. R. 1950 Some exact solutions of the flow through annular cascade actuator discs. J. Aeronaut. Sci. 17, 243-249.

Brown, G. L. \& Lopez, J. M. 1990 Axisymmetric vortex breakdown. Part 2. Physical mechanisms. J. Fluid Mech. 221, 553-576.

DAVIDSON, P. A. 1989 The interaction between swirling and recirculating velocity components in unsteady, inviscid flow. J. Fluid Mech. 209, 35-55.

Davis, S. H. 1976 The stability of time-periodic flows. Ann. Rev. Fluid Mech. 8, 57-74.

Greenspan, H. P. 1968 The Theory of Rotating Fluids. Cambridge University Press.

Kármán, TH. von 1921 Über laminare und turbulente Reibung. Z. Angew. Math. Mech. 1, 233-252.

Khalili, A. \& Rath, H. J. 1994 Analytical solution for a steady flow of enclosed rotating disks. $Z$. Angew Math. Phys. 45, 670-680.

Lopez, J. M. 1990 Axisymmetric vortex breakdown. Part 1. Confined swirling flow. J. Fluid Mech. 221, 533-552. 
Lopez, J. M. \& Perry, A. D. 1992 Axisymmetric vortex breakdown. Part 3. Onset of periodic flow and chaotic advection. J. Fluid Mech. 234, 449-471.

Mathis, D. M. \& Neitzel, G. P. 1985 Experiments on impulsive spin-down to rest. Phys. Fluids 28 , $449-454$.

MAXWORTHY, T. 1971 A simple observational technique for the investigation of boundary-layer stability and turbulence. In Turbulence Measurements in Liquids (ed. G. K. Paterson \& J. L. Zakin), pp. 32-37. Dept. Chemical Engineering, University of Missouri-Rolla.

Moore, F. K. 1956 Three-dimensional boundary layer theory. Adv. Appl. Mech. 4, 159-228.

Neitzel, G. P. \& Davis, S. H. 1980 Energy stability theory of decelerating swirl flows. Phys. Fluids 23, $432-437$.

Neitzel, G. P. \& Davis, S. H. 1981 Centrifugal instabilities during spin-down to rest in finite cylinders. Numerical experiments. J. Fluid Mech. 102, 329-352.

Rogers, M. H. \& LANCE, G. N. 1964 The boundary layer on a disc of finite radius in a rotating fluid. Q. J. Mech. Appl. Maths 17, 319-330.

RotT, N. \& Lewellen, W. S. 1967 Boundary layers and their interactions in rotating flows. Prog. Aeronaut. Sci. 7, 111-144.

SAVAŞ, Ö. 1983 Circular waves on a stationary disk in rotating flow. Phys. Fluids 26, 3445-3448.

SAVAŞ, Ö. 1985 On flow visualization using reflective flakes. J. Fluid Mech. 152, 235-248.

SAVAŞ, Ö. 1987 Stability of Bödewadt flow. J. Fluid Mech. 183, 77-94.

Schwiderski, E. W. \& LuGT, H. J. 1964 Rotating flows of von Kármán and Bödewadt. Phys. Fluids $7,867-875$.

SwEET, R. A. 1974 A generalized cyclic reduction algorithm. SIAM J. Num. Anal. 10, 506-592.

VALENTINE, D. T. \& MilleR, K. D. 1994 Generation of ring vortices in axisymmetric spin-down: A numerical investigation. Phys. Fluids 6, 1535-1547.

Weidman, P. D. $1976 a$ On the spin-up and spin-down of a rotating fluid. Part 1. Extending the Wedemeyer model. J. Fluid Mech. 77, 685-708.

WeIdMAN, P. D. $1976 b$ On the spin-up and spin-down of a rotating fluid. Part 2. Measurements and stability. J. Fluid Mech. 77, 709-735.

Weidman, P. D. 1989 Measurement techniques in laboratory rotating flows. In Advances in Fluid Mechanics Measurements (ed. M. Gad-el-Hak). Lecture Notes in Engineering, vol. 45, pp. 401534. Springer. 